\title{
Fundamental Tradeoff between Emission Intensity and Efficiency in Light-Emitting Electrochemical Cells
}

\author{
Stephan van Reenen, Rene A. J. Janssen and Martijn Kemerink
}

Linköping University Post Print

Tweet

N.B.: When citing this work, cite the original article.

Original Publication:

Stephan van Reenen, Rene A. J. Janssen and Martijn Kemerink, Fundamental Tradeoff between Emission Intensity and Efficiency in Light-Emitting Electrochemical Cells, 2015, Advanced Functional Materials, (25), 20, 3066-3073.

http://dx.doi.org/10.1002/adfm.201403945

Copyright: Wiley http://eu.wiley.com/WileyCDA/

Postprint available at: Linköping University Electronic Press

http://urn.kb.se/resolve?urn=urn:nbn:se:liu:diva-119585 


\section{WILEY-VCH}

DOI: 10.1002/ ((please add manuscript number))

Article type: Full Paper

\section{Fundamental tradeoff between emission intensity and efficiency in light-emitting electrochemical cells}

Stephan van Reenen, René A. J. Janssen, and Martijn Kemerink*

S. van Reenen, Prof. R. A. J. Janssen, Prof. M. Kemerink

Department of Applied Physics, Eindhoven University of Technology, P.O. Box 513, 5600

MB Eindhoven, The Netherlands

E-mail: martijn.kemerink@liu.se

Prof. M. Kemerink

Department of Physics, Chemistry and Biology (IFM), Linköping University, SE-58 183, Linköping, Sweden

Prof. R. A. J. Janssen

Department of Chemical Engineering, Eindhoven University of Technology, P.O. Box 513, 5600 MB Eindhoven, The Netherlands

Keywords: Organic Light-Emitting Electrochemical Cells, Recombination, Charge Transport, Conjugated Polymers, Doping

The characteristic doping process in polymer light-emitting electrochemical cells (LECs) causes a tradeoff between luminescence intensity and efficiency. Experiments and numerical modeling on thin film polymer LECs show that on the one hand carrier injection and transport benefit from electrochemical doping, leading to increased electron-hole recombination. On the other hand the radiative recombination efficiency is reduced by exciton quenching by polarons involved in the doping. Consequently the quasi-steady-state luminescent efficiency decreases with increasing ion concentration. The transient of the luminescent efficiency shows a characteristic roll-off while the current continuously increases, attributed to ongoing electrochemical doping and the associated exciton quenching. Both effects can be modeled by exciton polaron-quenching via diffusion-assisted Förster resonance energy transfer. These results indicate that the tradeoff between efficiency and intensity is fundamental, suggesting that the application realm of future LECs should be sought in high-brightness, low-production cost devices, rather than in high-efficiency devices. 


\section{WILEY-VCH}

\section{Introduction}

The unique selling point of light-emitting electrochemical cells (LECs) is the fact that a single active layer that enables both ionic and electronic conduction can perform the tasks of injection, transport, and recombination of electrons and holes. ${ }^{[1,2]}$ Upon application of a sufficiently large electrical bias ions and electronic charges redistribute to effectively form a five-layer device with injection and doped transport layers for electrons and holes sandwiching an intrinsic recombination zone. ${ }^{[2,3]}$ Despite significant efforts aimed at improving the performance and the understanding of LECs, their luminous efficiency has remained moderate $\left(20 \mathrm{~lm} \mathrm{~W}^{-1}\right.$ for best reported white $\left.\mathrm{LEC}^{[4]}\right)$ compared to multilayer organic light emitting diodes (OLEDs) $\left(114 \mathrm{~lm} \mathrm{~W}^{-1}\right.$ for best reported white OLED $\left.{ }^{[5]}\right)$. In view of the well-established excellent injection and transport characteristics of LECs this is surprising. To determine and possibly remove the origin of the limited performance of LECs, the conversion from electrical current into radiatively decaying excitons must be fully understood.

The current-to-light conversion occurs through multiple steps, starting from recombination of carriers into excitons, followed by exciton decay, and outcoupling of the generated light. As for the first step, the presence in well-functioning LECs of a stable intrinsic recombination zone between high-density p- and n-type doping zones ${ }^{[2,3]}$ ensures balanced hole and electron currents $^{[6]}$ and a close to unity recombination efficiency. ${ }^{[7]}$ As for the last step, the light outcoupling in LECs is expected to be similar to that in OLEDs. Hence the relatively low efficiency of LECs compared to OLEDs is most likely related to nonradiative exciton decay.

Indeed, the radiative efficiency of the operational LECs remains typically well below the (fine) PL efficiency of the emissive species used, both for LECs based on polymers ${ }^{[8]}$ and on ionic transition metal complexes (iTMCs) ${ }^{[7]}$. Various non-radiative decay processes can in principle be responsible for this. Quenching of excitons may be induced by the large density of polarons in the doped regions. ${ }^{[9]}$ Additionally, exciton-exciton interactions may be 


\section{WILEY-VCH}

expected as a high concentration of excitons is formed in the relatively narrow intrinsic zone. Also impurities in the active layer, e.g. related to the electrolyte, may give rise to further reduction of the radiative fraction.

A strong indication that excitons are quenched by polarons in LECs is the typical roll-off in efficiency for both polymer- and iTMC-based LECs. ${ }^{[10]}$ This roll-off occurs while the current density continues to increase, which indicates continuous electrochemical doping. This slow doping process was previously attributed to slow dissociation of salt into mobile ions that subsequently can be used in doping. ${ }^{[3]}$ In fact, in a number of recent papers doping-related quenching was proposed as cause for the efficiency roll-off. ${ }^{[11,12,13]}$ Formal justification of this assignment is however lacking.

Here we investigate the difference in efficiency in LECs compared to OLEDs based on the same organic semiconductor. The origin of the efficiency roll-off in LECs is studied in detail as well as the influence of the admixed salt concentration $c 0$ in the active layer on the quasisteady-state electroluminescent efficiency. Experiments show a strong reduction in quasisteady-state efficiency for increasing $c_{0}$. Additionally, transient characterization of these devices displays the typical roll-off in efficiency during electrochemical doping. The data is reproduced by numerical modeling to constitute that, compared to OLEDs, the efficiency in LECs is limited by polaron-induced exciton quenching. These results point towards a fundamental tradeoff in LECs between charge transport, with more doping giving rise to higher current densities and even higher emission intensities, and radiative decay, with polarons involved in the doping opening a non-radiative decay channel.

\section{Results and Discussion}

The luminescent efficiency of polymer LECs is experimentally investigated by measurement of the transient luminescent efficiency and quasi-steady-state luminescent efficiency in devices with varying doping concentrations. The doping concentration is altered by variation 


\section{WILEY-VCH}

of the admixed salt density $c_{0}$. Simulations to complement the experiments were performed by a numerical drift-diffusion model. Details are described in the Experimental section and section A of the Supporting Information.

\subsection{Steady-state operation}

To avoid studying injection limited devices at low $c_{0}$, LECs were prepared with low work function cathodes, LiF/Al, and high work function anodes, ITO/PEDOT:PSS, to enable ohmic injection in the semiconductor, a phenyl-substituted poly( $p$-phenylene vinylene) copolymer commonly termed "Super Yellow" (SY-PPV). The (limited) effect of using different electrode materials is shown in the supplemental information section B. The active layer consisted of SY-PPV: Poly(ethylene oxide) (PEO) in a fixed weight ratio of 5:1 and a variable amount $x$ (for $x=2 \cdot 10^{-4}-0.6$ ) of salt, $\mathrm{KCF}_{3} \mathrm{SO}_{3}$, resulting in an average salt density between $\sim 10^{24}$ and $\sim 10^{27} \mathrm{~m}^{-3}$. The salt density was determined from these weight ratios, the molecular mass of the constituents and assuming a density of $1 \mathrm{~g} \mathrm{~cm}^{-3}$ in the active layer. Several series of LECs were studied with varying active layer thickness $L$ of $100 \pm 10,250 \pm 25$ and $600 \pm 40 \mathrm{~nm}$.

The latter two series were prepared by spin-coating subsequent layers on top of each other. ${ }^{[14]}$ Care was taken that any previous layers were dry before depositing the next layer. The devices were electrically characterized by subsequent voltage sweeps up to $8 \mathrm{~V}$. During these sweeps, the current density was found to converge to a single quasi-steady-state value at $8 \mathrm{~V}$. Similar voltage sweeps up to lower bias voltages resulted in hysteresis in subsequent sweeps, i.e. a continuous increase in the current density at the maximum bias voltage, indicating that a quasi-steady-state situation had not been reached. For the $8 \mathrm{~V}$ scans, electrochemical sidereactions were not observed by eye, nor was there any indication of this in the relatively fast $\mathrm{I}-\mathrm{V}$ sweeps as the current density at $8 \mathrm{~V}$ was not observed to decrease for subsequent scans. For I-V sweeps with a maximal voltage above $8 \mathrm{~V}$, such reductions in current density were observed. Prolonged operation at $8 \mathrm{~V}$, unlike the I-V sweeps mentioned here, did however 


\section{WILEY-VCH}

also result in degradation of the device. The current density and luminance at $8 \mathrm{~V}$ are plotted for all three series in Figure 1a, $\mathbf{b}$.

To enable qualitative comparison with the experiments, the results of simulations of the steady-state current density and luminance of an LEC biased at $3.5 \mathrm{~V}$ are shown in Figure 1a and b. Here $L$ is $100 \mathrm{~nm}$ and a constant electronic carrier mobility is assumed. A constant mobility was taken to keep the calculation time within reasonable limits and to avoid the addition of too many parameters. Addition of such a doping dependent mobility would lead to an even stronger enhancement of the current as a function of doping density than the enhancement shown in Figure 1a. Due to long calculation times, only a single device thickness $(L)$ was modeled. Given the constant electronic carrier mobility, variation of $L$ between 100 and $600 \mathrm{~nm}$ would mainly result in enlargement of the doped regions and the supply of carriers towards the recombination zone would remain similar. Therefore, only minor quantitative differences are expected upon varying $L$. A diffusion-assisted polaroninduced exciton-quenching model was implemented in the model as described in the Experimental section. The following parameters related to the excitons were set to values obtained from literature: the radiative decay rate $k_{\text {decay }}=1 / \tau_{D}^{0}=1.54 \mathrm{~ns}^{-1}$ found in SY-PPV and the exciton diffusion coefficient $D_{\mathrm{ex}}=3 \cdot 10^{-8} \mathrm{~m}^{2} \mathrm{~s}^{-1}$ found in the related NRS-PPV. ${ }^{[15,16]}$ The Förster critical radius $R_{0}$ was set at $3 \mathrm{~nm}$. This value is similar to the values obtained in Ref. $\left[{ }^{9}\right]$ for p- and n-type doping of SY-PPV of $2.8 \pm 0.6$ and $2.3 \pm 0.4 \mathrm{~nm}$, respectively. These were determined from the polaron absorption and excitation spectra.

As expected, the simulations show that an enhancement of the salt density results in an enhancement of the current density. The luminance increases accordingly. This is related to the ability of the device to prevent space charge formation while accumulating larger densities of mobile charge carriers in the active layer through electrochemical doping. ${ }^{[12,17]}$ For the thicker LECs $(L=250$ and $600 \mathrm{~nm})$ an enhancement of the current density is also experimentally observed for increasing salt density. For the $600 \mathrm{~nm}$ device the luminance is 


\section{WILEY-VCH}

also observed to be enhanced. No luminance measurements were performed for the $250 \mathrm{~nm}$ devices. For the series with $L=100 \mathrm{~nm}$, surprisingly a current density is observed that is nearly independent of the admixed salt density. Apparently carrier transport cannot be further enhanced in these $100 \mathrm{~nm}$ thick LECs, probably due to a cancelation of various factors. The luminance is however reduced, which is rationalized below.

The efficiency of the experimental and simulated LECs (see Figure 1) is plotted in Figure 2. The dashed lines indicate the experimentally determined efficiency of OLEDs with layer stacks ITO/PEDOT:PSS/SY-PPV/LiF/Al and ITO/PEDOT:PSS/SY-PPV:PEO (5:1 in w\%)/LiF/Al. The efficiency of these OLEDs is highly similar, indicating that the inclusion of PEO hardly affects the efficiency. It is also similar to the efficiencies measured in the LECs at relatively low $c_{0}$. The efficiency of the 100 and $600 \mathrm{~nm}$ thick LECs in Figure 2 (symbols) is reduced by an order of magnitude by increasing the salt density from $\sim 10^{24}$ to $\sim 10^{27} \mathrm{~m}^{-3}$.

In the numerical modeling, excitons can either decay radiatively or be quenched by electrons or holes. The efficiency of the modeled LEC is plotted in Figure 2 for varying exciton diffusion coefficients $\left(D_{\mathrm{ex}}=3 \cdot 10^{-8} \mathrm{~m}^{2} \mathrm{~s}^{-1}\right.$ was experimentally determined in NRS-PPV, which is structurally and electro-optically comparable to SY-PPV). ${ }^{16}$ The modeled results (in red in Figure 2) lie more or less in between the experimentally obtained efficiency values (symbols in Figure 2). The maximal efficiency of the model ( 0.25 photon/electron) is set at the same position on the vertical axis as the experimentally determined efficiency of the salt-free OLEDs (dashed lines). This procedure is used to effectively account for the absence of other non-radiative loss channels and outcoupling effects in our simulations. Importantly, the same $\mathrm{y}$-axis scale on left and right assures that relative salt-induced changes can be one-on-one compared.

The qualitative and quantitative agreement between model and experiment strongly suggests that polaron-induced exciton quenching can indeed dominate the efficiency reduction in polymer LECs. Note that all relevant parameters in the model have been independently 


\section{WILEY-VCH}

determined or were taken from literature, so that the agreement does not rely on any fitting. On top of polaron-induced exciton quenching also doping-induced self-absorption has recently been shown to lead to a reduced output of electroluminescence in LECs during operation. ${ }^{[13]}$

\subsection{Transient operation}

Another route to determine the origin of the reduced luminescent efficiency in LECs is by study of the transient behavior. As already shown for different types of LECs, ${ }^{[10]}$ after application of a constant, above-band gap bias voltage the LECs show a characteristic roll-off of the efficiency while the current density continues to increase. In the LECs studied here this characteristic is also observed as shown in Figure 3. The current, luminance, and efficiency were characterized in three subsequent measurements on different timescales as shown in Figure $3 b$. Due to the disabling of the bias voltage in between, the transients do not connect perfectly, but they do show the general behavior of the LEC over eight orders in time. Transients below $\sim 10^{0}$ seconds were observed to be qualitatively reproducible with the same LEC, as deduced from repetitive measurements. Quantitatively minor differences occur nevertheless as visible in Figure 3b. Prolonged operation for $t>10^{1} \mathrm{~s}$ however typically changes the LECs irreversibly, presumably due to remnant doping which does not relax within at least several hours.

Also in transient experiments, enhancement of $c_{0}$ results in enhanced quenching as shown in

Figure 4. Like in Figure 1 the current density shows a clearly increasing trend with increasing $c 0$, at the cost of efficiency. Roll-off in the efficiency transients is observed for $c_{0}>10^{25} \mathrm{~m}^{-3}$, with lower (near) saturation efficiencies for larger $c_{0}$. The relatively low efficiency for the devices with a low $c_{0}$, i.e. $1 \cdot 10^{24}$ and $3 \cdot 10^{24}$ can be attributed to a relatively long stabilization time compared to devices with higher values of $c_{0}$. 


\section{WILEY-VCH}

To complement the experiments, the numerical model was used to calculate current, luminance (see section C of the Supporting Information), and efficiency transients in LECs. In case only free ions are considered in the model the transients represented by the dashed lines in Figure 5b and d are obtained. Neither a large transient enhancement in current density nor a roll-off in efficiency is observed. Although the current density does increase for $10^{-4}<t$ (s) $<2 \cdot 10^{-3}$, the timescale on which this happens is very short compared to transients shown in Figure 3b. Clearly a key ingredient is missing in this description of the LECs. This transient however does resemble the transients typically observed in planar LECs as shown for example in Ref. $\left[{ }^{2}\right]$.

In Ref. $\left[{ }^{3}\right]$ we found that the inclusion of anion-cation pairs, i.e. salt, with a binding energy $E_{\mathrm{s}}$ enables a good description of the transient behavior of the electronic current density in thin film LECs. Next to the binding energy, also the capture coefficient $\gamma_{c}$ of anions and cations is a free parameter as described in section A.4 of the Supporting Information. Introduction of bound anion-cation pairs in the model used here significantly changes the transients as demonstrated in Figure 5 for varying $E_{\mathrm{s}}$ and $\gamma_{c}$. The transients in Figure 5 are from LECs which at $t=0$ are in equilibrium regarding salt association and dissociation. $E_{\mathrm{s}}$ therefore determines the initial fraction of mobile ions of the total salt density $c_{0}$. The turn-on transients of LECs with varied $E_{\mathrm{s}}\left(\gamma_{c}\right.$ was changed so as to keep the salt dissociation coefficient $\gamma_{d}=$ $\gamma_{c} \cdot \exp \left(E_{\mathrm{S}} / k T\right)$ constant $)$ is shown in Figure 5a and c. They show, as expected, that for a large initial mobile ion fraction, the initial current is relatively large. Turn-on of light emission is observed around $t \sim 10^{-4} \mathrm{~s}$ which indicates that the first electrons and holes have traversed the device. This time is related to the carrier mobility and the externally applied electric field. Simultaneously electrochemical doping takes place by association of free charges with mobile ions. Dissociation of bound anion-cation pairs takes place as well, enabling further electrochemical doping. As salt dissociation is the slowest process, it limits the evolution of the turn-on transient. Simulations for different capture coefficients $\gamma_{c}$ and a fixed $E_{\mathrm{s}}=0.54 \mathrm{eV}$ 


\section{WILEY-VCH}

are shown in Figure $5 \mathrm{~b}$ and d. Reduction of $\gamma_{c}$ mainly slows down the doping process and enhances the time needed to reach steady-state accordingly. Due to long calculation times steady-state was not reached in these simulations, but the transients should converge to the same final state. The reason is that at steady-state all ions are completely separated from each other and have a fixed density profile due to the contacts which block ion transport. As a consequence not the mobility, the binding energy or the capture coefficient, but only the density of ions is of importance for steady-state operation of the modeled LEC. ${ }^{[2]}$

Looking at the transient efficiency of the calculated LECs in Figure 5c and d, a slow roll-off is observed on the same timescale as the current increase -both result from the slow ion dissociation enabling the ongoing doping process. In Figure 6 the simulation for $E_{\mathrm{s}}=0.42 \mathrm{eV}$ and $\gamma_{c}=3 \cdot 10^{-17} \mathrm{~m}^{3} \mathrm{~s}^{-1}$ is plotted on a linear and logarithmic scale. Qualitatively the experimental and calculated transients in Figure 3 and Figure 6 display the same features. In the modeling (experimental) results, the onset of luminance is observed at $10^{-4} \mathrm{~s}\left(10^{-3} \mathrm{~s}\right)$ followed by a relatively constant current density until $10^{-2} \mathrm{~s}\left(10^{1} \mathrm{~s}\right)$. Current density and luminance subsequently continue to increase, whereas the efficiency reaches its peak-value and rolls off.

In the experiment (Figure 3) additional steps in the efficiency and luminance are observed between $10^{-3} \mathrm{~s}<t<10^{3} \mathrm{~s}$ before the maximal efficiency is reached, which are not reproduced by the numerical model. The first step at $\sim 10^{-1} \mathrm{~s}$ may be related to initial imbalanced charge transport due to a difference in electron and hole mobility, resulting in recombination near one of the contacts followed by exciton quenching due to the contact. In the model equal carrier mobilities were chosen. Note that after significant electrochemical doping the recombination efficiency is expected to be $\sim 100 \%$ and recombination is expected to take place further away from the contacts. The increase in efficiency at $\sim 10^{2} \mathrm{~s}$ is possibly related to a reduced importance of non-radiative trap-assisted recombination. ${ }^{18}$ At relatively high current densities, 


\section{WILEY-VCH}

these traps are saturated so their relative effect on recombination is reduced. Consequently the efficiency is raised at higher current densities. This may also explain why the efficiency is reduced for the lowest ion densities in Figure 2 and 4b. Trap-related non-radiative exciton decay processes were not taken into account by the model to not obscure the calculated trends due to polaron-induced exciton quenching.

The transients in Figure 6 are qualitatively similar to the transients in Figure 3. For a more quantitative agreement some parameters can be modified. The Supporting Information (section D) discusses in detail how these parameters were chosen and their relation to experiments.

Due to extremely long calculation times a simulation with the expectedly optimal parameters could not be performed. However, for $\mu_{\text {ion }}=10^{-12}$ instead of $\sim 10^{-15} \mathrm{~m}^{2} \mathrm{~V}^{-1} \mathrm{~s}^{-1}$ a simulation was carried out, shown in Figure 7 as lines. Note that the numerical model does not take into account the radiative efficiency related to the non-unity PL quantum yield of SY-PPV, which is $17 \%,{ }^{[15]}$ nor the out-coupling efficiency which is approximately $20 \%$ in the experiment. Therefore, to enable good comparison with the experiments, the luminance and the efficiency are multiplied by $0.2 \cdot 0.17$. The experimental results from Figure 3 are included as symbols. The experimental data related to the luminance and the efficiency were converted from $\mathrm{cd} \mathrm{m}^{-2}$ and $\mathrm{cd} \mathrm{A}^{-1}$ to photon $\mathrm{m}^{-2} \mathrm{~s}^{-1}$ and photon per electron, respectively, by two related conversion factors. The conversion factor from $\mathrm{cd} \mathrm{m}^{-2}$ to photon $\mathrm{m}^{-2} \mathrm{~s}^{-1}$ is derived in section $\mathrm{E}$ of the Supporting Information and was found to be $3.5 \cdot 10^{16}$ photon $\mathrm{cd}^{-1} \mathrm{~s}^{-1}$. From this value the conversion factor from $\mathrm{cd} \mathrm{A}^{-1}$ to photon per electron follows as 0.0056 photon $\mathrm{A} \mathrm{cd}^{-1}$ per electron. To enable comparison of the transients during electrochemical doping in model and experiment, the horizontal axis of the modeled results was shifted to compensate for the enhanced ion mobility used in the model. Here we found that the scaling factor is approximately $2 \cdot 10^{3}$ which means that, based on the fit, the ion mobility in the experiment is roughly $5 \cdot 10^{-16} \mathrm{~m}^{2} \mathrm{~V}^{-1} \mathrm{~s}^{-1}$. Electrochemical doping in Figure 7 takes place at $10^{-1}<t<10^{4}$. 


\section{WILEY-VCH}

During this time, the current, luminance and efficiency transients show quantitative agreement between model and experiment within an order of magnitude. Given the complexity of the system we consider this very satisfactory.

The results of the transient (see Figure 7) and steady-state (see Figure 1 and 2) operation of polymer LECs has shown that polaron-induced exciton quenching can be considered as one of the main processes which result in a reduction of the electroluminescent efficiency. Requirements for effective polaron-induced exciton quenching are i) exciton formation near a high density of polarons, ii) exciton diffusion, iii) spectral overlap for exciton emission and polaron absorption, and iv) a sufficient exciton lifetime. ${ }^{[9,19]}$ To avoid exciton quenching, one or more of these requirements should not be fulfilled. Modification of the properties of the conjugated polymer is challenging as it should then be optimized simultaneously for both charge transport and luminescent efficiency, at the required band gap. To circumvent this, for example dyes can be considered. Qian et al. ${ }^{[20]}$ recently reported on a quantum dot LEC reaching a similar luminescent efficiency as a multilayer quantum dot OLED, despite the presence of some efficiency roll off. Further optimization of such systems may lead to efficient, single layered electroluminescent devices, combining the high efficiencies of OLEDs and the easy processing of LECs.

\section{Conclusion}

The emission intensity increase and efficiency reduction in polymer LECs due to electrochemical doping have been systematically studied in experiments by investigation of the transient and quasi-steady-state performance. Enhancement of the salt density in polymer LECs is found to reduce the efficiency for admixed salt densities $>10^{25} \mathrm{~m}^{-3}$. This reduction in efficiency was successfully reproduced by numerical modeling of LECs in which the only non-radiative loss channel of excitons was quenching by polarons involved in doping. Also the transient efficiency roll-off, typical for many types of LECs, was reproduced by the model. 


\section{WILEY-VCH}

The roll-off is caused by ongoing doping that is enabled by the slow dissociation of salt into mobile ions that subsequently are used to further dope the semiconductor. Hence, the current continues to increase while the efficiency rolls off.

The results show that the ions which enable electrochemical doping improve electrical transport and concomitantly electron-hole recombination in polymer LECs at the cost of the luminescent efficiency. This tradeoff seems fundamental in nature, suggesting that the application realm of future LECs should be sought in high-brightness, low-production cost devices, rather than in high-efficiency devices.

\section{Experimental Section}

Device preparation: The conjugated polymer used in the active layer of the sandwich LECs is a phenyl-substituted poly(p-phenylene vinylene) copolymer (SY-PPV, Merck, catalogue number PDY-132), commonly termed "Super Yellow". Poly(ethylene oxide) (PEO, $M_{\mathrm{w}}=$ $5 \cdot 10^{5} \mathrm{~g} \mathrm{~mol}^{-1}$, Aldrich) was used as received. The salt potassium trifluoromethanesulfonate $\left(\mathrm{KCF}_{3} \mathrm{SO}_{3}, 98 \%\right.$, Aldrich) was dried at a temperature $T=373 \mathrm{~K}$ under vacuum before use. SY-PPV $\left(5 \mathrm{mg} \mathrm{ml}^{-1}\right)$, PEO $\left(10 \mathrm{mg} \mathrm{ml}^{-1}\right)$, and $\mathrm{KCF}_{3} \mathrm{SO}_{3}\left(20 \mathrm{mg} \mathrm{ml}^{-1}\right)$ were dissolved in cyclohexanone (> $99 \%$, anhydrous, Aldrich). These solutions were mixed together in varying weight ratios. The resultant stock solutions were thereafter stirred on a magnetic hot plate at $T$ $=323 \mathrm{~K}$ for at least $5 \mathrm{~h}$.

Cleaned glass-ITO substrates were first spin coated with poly(3,4-ethylenedioxythiophene)poly(styrenesulfonate) (PEDOT:PSS, Clevios P VP AI 4083) from Heraeus (at 2000 RPM for $60 \mathrm{~s})$ and subsequently transferred to a glove box under $\mathrm{N}_{2}$ atmosphere $\left(\left[\mathrm{O}_{2}\right]<1 \mathrm{ppm}\right.$ and $\left.\left[\mathrm{H}_{2} \mathrm{O}\right]<1 \mathrm{ppm}\right)$ where the substrates were annealed for 10 minutes at $120{ }^{\circ} \mathrm{C}$. All the next steps were done in a glove box. Subsequently the stock solution was spin coated (in general at $1000 \mathrm{RPM}$ for $60 \mathrm{~s}$ ) after which the samples were dried at $T=323 \mathrm{~K}$ for at least $2 \mathrm{~h}$ on a hot plate. Then the substrates were transferred to a thermal evaporator where $\mathrm{LiF} / \mathrm{Al}$ or $\mathrm{Al}$ 


\section{WILEY-VCH}

electrodes were deposited by thermal evaporation under high vacuum ( $p \sim 10^{-6}$ mbar) on top of the spin-coated films. The active areas of the devices, defined by a shadow mask, were either 0.091 or $0.161 \mathrm{~cm}^{2}$. The thickness of the active layers was measured after complete electrical characterization by profilometry in air.

Electrical characterization: Current-luminance-voltage characteristics were measured by a Keithley 2636a sourcemeter and a silicon photodiode. The brightness was calibrated with a luminance meter (LS-110 Konica-Minolta). In general two types of measurements were performed. Current and luminance were measured while sweeping the bias voltage $V_{\text {bias. }}$ To achieve reproducible $I$ - $V$ curves, 3 subsequent voltage sweeps were performed typically from $0 \mathrm{~V}$ to $8 \mathrm{~V}$ to $0 \mathrm{~V}$ in $\sim 20 \mathrm{~s}$. In addition, the current and luminance were also measured as a function of time at a constant bias voltage of $6 \mathrm{~V}$. To achieve the short-timescale measurement as shown in Figure $3 \mathrm{~b}$ (for $t<10^{-1} \mathrm{~s}$ ), the Analog/Digital converter speed of the Keithley 2636a sourcemeter was reduced to 0.001 number of power line cycles. Consequently there is slightly more noise in the signal.

Computational details: For the numerical simulations, a 2-dimensional drift-diffusion model was used $^{[21]}$ which is described in detail in section A of the Supporting Information. Here follows a general description of the used parameters. Deviating parameters are mentioned where necessary. The model was used to describe a 1-dimensional device with constant gridpoint spacing of $2 \mathrm{~nm}$ and a total active layer thickness of $100 \mathrm{~nm}$. The carrier mobility was chosen to be field and doping independent and set to $\mu_{\mathrm{p} / \mathrm{n}}=10^{-11} \mathrm{~m}^{2} \mathrm{~V}^{-1} \mathrm{~s}^{-1}$ unless stated otherwise. The anion and cation mobilities were chosen to be $\mu_{\text {ion }}=10^{-12} \mathrm{~m}^{2} \mathrm{~V}^{-1} \mathrm{~s}^{-1}$. The ion mobility at the interface layer at the contacts was set to zero to prevent accumulation at the electrodes. If not, an unphysically large number of ions would pile up next to the electrodes and be completely screened by the metal electrode. This is a crude but sufficient way of introducing a maximum doping density due to finite ion size effects. The temperature is set at $300 \mathrm{~K}$, the relative dielectric constant at 4 . A semiconductor band gap of $2.4 \mathrm{eV}$ was chosen 


\section{WILEY-VCH}

and ohmic and non-ohmic injection was modeled by setting the electron and hole injection barriers to 0.1 and $1.2 \mathrm{eV}$, respectively. The applied bias voltage is $3.5 \mathrm{~V}$. The initial homogeneous salt density was set at varying values ranging from $10^{22}$ to $3 \cdot 10^{26} \mathrm{~nm}^{-3}$. Optionally a binding energy between the ions was introduced as explained in section A.4 of the Supporting Information with varying values for the binding energy $E_{\mathrm{s}}$ and the capture coefficient $\gamma_{c}\left(\mathrm{~m}^{3} \mathrm{~s}^{-1}\right)$. Optionally a doping dependent mobility as described in section A.3 of the Supporting Information was used with parameters which were obtained after fitting the doping dependent mobility measured in SY-PPV reported in Ref. $\left[{ }^{9}\right]$.

Exciton quenching by polarons is described by diffusion-mediated Förster resonance energy transfer. ${ }^{[9]}$ Implementation of this model is as follows. For each time step an exciton quenching rate is calculated at each grid-point for a given polaron density on the same gridpoint. Diffusion of excitons between grid points is included. Diffusion within a grid-point is taken into account via the implemented exciton quenching rate. The polaron density dependent singlet exciton quenching rate $k_{\mathrm{PQ}}\left(\mathrm{m}^{-3} \mathrm{~s}^{-1}\right)$ is:

$$
k_{P Q}=\frac{\left[1-\exp \left(4 \pi R_{e f f} D_{e x c} C_{A} t_{0}-2 \cdot 4 \sqrt{\pi D_{e x c}} R_{e f f}^{2} C_{A} \sqrt{t_{0}}\right)\right]}{t_{\text {step }}},
$$

where $R$ eff is an effective quenching radius based on the Förster critical radius for quenching, $R_{0}$, the exciton lifetime in absence of any quenching, $\tau_{D}^{0}$, and exciton diffusion with diffusion constant $D_{\text {ex }}$ as described by ${ }^{19}$

$$
R_{e f f}=0.676\left(\frac{R_{0}^{6}}{\tau_{D}^{0} D_{e x}}\right)^{1 / 4}
$$

$C_{\mathrm{A}}$ is the acceptor concentration, which is set equal to the sum of the electron and hole densities, and $t_{0}$ is the time step taken in the calculation. Note that polarons on neighboring grid-points do not contribute to exciton quenching, which will give rise to some underestimation of the quenching rate. 


\section{WILEY-VCH}

The computational time to reach quasi-steady-state depends on the number of time steps taken. In simulating LEC transients, the transient length is determined by the slowest process, ion redistribution, whereas the maximum time step is determined by the fastest process, related to exciton diffusion and decay. To obtain a reasonable computational time, which is proportional to the ratio of the transient length and the time step, we needed to slow down the fastest processes. The radiative decay rate of the excitons, $k_{F L}$, and $D_{\text {ex }}$ were reduced by a factor $10^{-5}$. The resultant delay could, however, give errors in the calculation of the transient behavior of the exciton decay processes. For transients which are limited by the movement of ions care is taken that the ion diffusion coefficient $D_{\text {ion }}=\mu_{\text {ion }} k T / q$ remains smaller than $D_{\text {ex }}$ by at least a factor 10 to minimize this effect.

\section{Supporting Information}

Supporting Information is available from the Wiley Online Library or from the author.

\section{Acknowledgements}

This work is supported by NanoNextNL, a micro and nanotechnology consortium of the Government of the Netherlands and 130 partners, Project No. NNNL.06D.04.

Received: ((will be filled in by the editorial staff))

Revised: ((will be filled in by the editorial staff)) Published online: ((will be filled in by the editorial staff))

[1] Q. B. Pei, G. Yu, C. Zhang, Y. Yang, A. J. Heeger, Science 1995, 269, 1086; Q. B. Pei, Y. Yang, G. Yu, C. Zhang, A. J. Heeger, J. Am. Chem. Soc. 1996, 118, 3922.

[2] S. van Reenen, P. Matyba, A. Dzwilewski, R. A. J. Janssen, L. Edman, M. Kemerink, J. Am. Chem. Soc. 2010, 132, 13776.

[3] S. van Reenen, R. A. J. Janssen, M. Kemerink, Adv. Funct. Mater. 2012, 22, 4547.

[4] T. Hu, L. He, L. Duan, Y. Qiu, J. Mater. Chem. 2012, 22, 4206; Y. P. Jhang, H. F. Chen, H. B. Wu, Y. S. Yeh, H. C. Su, K. T. Wong, Org. Electron. 2013, 14, 2424.

[5] http://panasonic.co.jp/corp/news/official.data/data.dir/2013/05/en130524-6/en130524$\underline{6 . h t m l}$

[6] M. J. Tsai, H. F. Meng, J. Appl. Phys. 2005, 97, 114502. 


\section{WILEY-VCH}

[7] H. J. Bolink, E. Coronado, R. D. Costa, N. Lardies, E. Orti, Inorg. Chem. 2008, 47, 9149.

[8] F. Marchioni, R. Chiechi, S. Patil, F. Wudl, Y. Chen, J. Shinar, Appl. Phys. Lett. 2006, 89, 061101.

[9] S. van Reenen, M. V. Vitorino, S. C. J. Meskers, R. A. J. Janssen, M. Kemerink, Phys. Rev. B 2014, 89, 205206.

[10] S. van Reenen, T. Akatsuka, D. Tordera, M. Kemerink, H. J. Bolink, J. Am. Chem. Soc. 2013, 135, 886.

[11] S. B. Meier, D. Hartmann, D. Tordera, H. J. Bolink, A. Winnacker, W. Sarfert, Phys. Chem. Chem. Phys. 2012, 14, 10886; X. Y. Li, J. Gao, G. J. Liu, Appl. Phys. Lett. 2013, 102, 223303.

[12] J. F. Fang, Y. L. Yang, L. Edman, Appl. Phys. Lett. 2008, 93, 063503; J. Fang, P. Matyba, N. D. Robinson, L. Edman, J. Am. Chem. Soc. 2008, 130, 4562.

[13] N. Kaihovirta, A. Asadpoordarvish, A. Sandstrom, L. Edman, Acs Photonics 2014, 1, 182.

[14] X. Y. Li, J. Gao, G. J. Liu, Org. Electron. 2013, 14, 2711.

[15] E. W. Snedden, L. A. Cury, K. N. Bourdakos, A. P. Monkman, Chem. Phys. Lett. 2010, 490, 76.

[16] D. E. Markov, J. C. Hummelen, P. W. M. Blom, A. B. Sieval, Phys. Rev. B 2005, 72, 045216.

[17] S. van Reenen, P. Matyba, A. Dzwilewski, R. A. J. Janssen, A. Edman, M. Kemerink, Adv. Funct. Mater. 2011, 21, 1795.

[18] M. Kuik, L. J. A. Koster, A. G. Dijkstra, G. A. H. Wetzelaer, P. W. M. Blom, Org. Electron. 2012, 13, 969.

[19] B. Valeur, Molecular Fluorescence: Principles and Applications, WILEY-VCH, Weinheim 2001.

[20] G. Qian, Y. Lin, G. Wantz, A. R. Davis, K. R. Carter, J. J. Watkins, Adv. Funct. Mater. 2014, 24, 4484.

[21] S. B. Meier, S. Van Reenen, B. Lefevre, D. Hartmann, H. J. Bolink, A. Winnacker, W. Sarfert, M. Kemerink, Adv. Funct. Mater. 2013, 23, 3531. 
WILEY-VCH

(a)

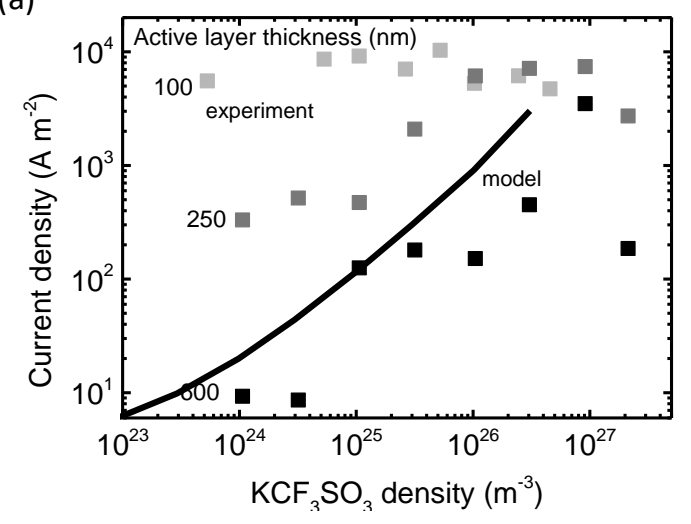

(b)

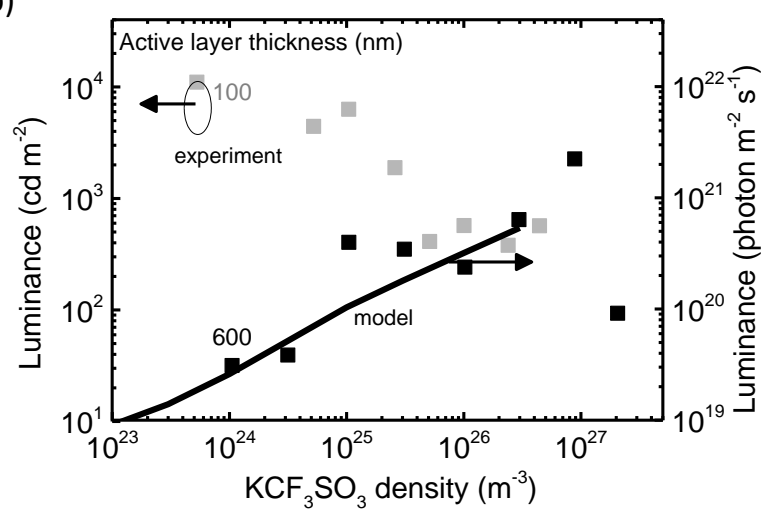

Figure 1. Experimentally (symbols, $V_{\text {bias }}=8 \mathrm{~V}$ ) and numerically (lines, $V_{\text {bias }}=3.5 \mathrm{~V}$ ) determined quasi-steady-state current density (a) and luminance (b) of LECs. The active layer thicknesses of the experimental ITO/PEDOT:PSS/SY-PPV:PEO: $\mathrm{KCF}_{3} \mathrm{SO}_{3}=5: 1: x / \mathrm{LiF} / \mathrm{Al}$ devices is indicated. No luminance measurements were performed on the $250 \mathrm{~nm}$ cells. The thickness of the modeled LEC was $100 \mathrm{~nm}$. 


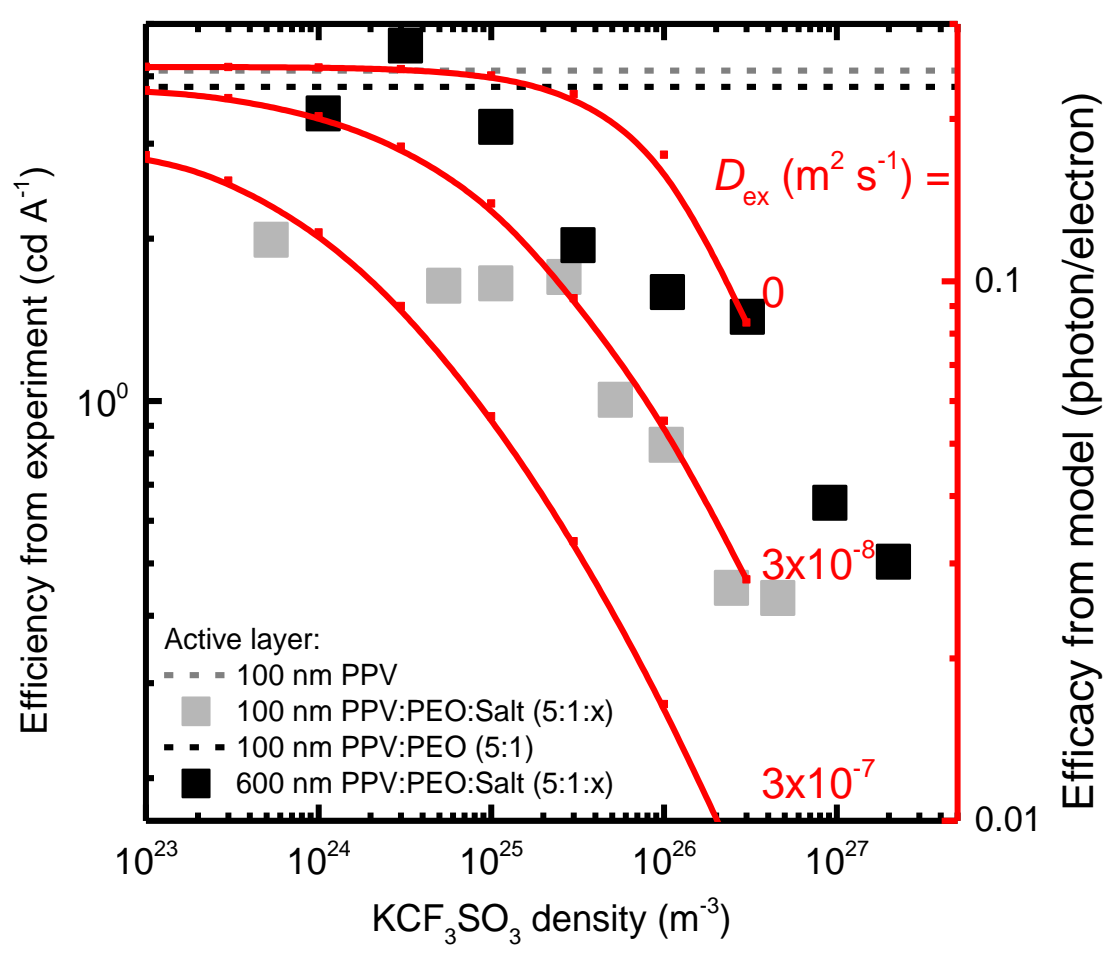

Figure 2. Experimentally (squares, $V_{\text {bias }}=8 \mathrm{~V}$ ) and numerically (red lines + symbols, $V_{\text {bias }}=$ $3.5 \mathrm{~V}$ ) determined efficiency in LECs vs. admixed $\mathrm{KCF}_{3} \mathrm{SO}_{3}$ density. The dashed lines indicate the efficiency in absence of $\mathrm{KCF}_{3} \mathrm{SO}_{3}$. The legend indicates the active layer composition for the experiments, which is sandwiched between ITO/PEDOT and LiF/Al electrodes. The exciton diffusion coefficients in $\mathrm{m}^{2} \mathrm{~s}^{-1}$ are indicated for each numerical calculation. 


\section{WILEY-VCH}

(a)

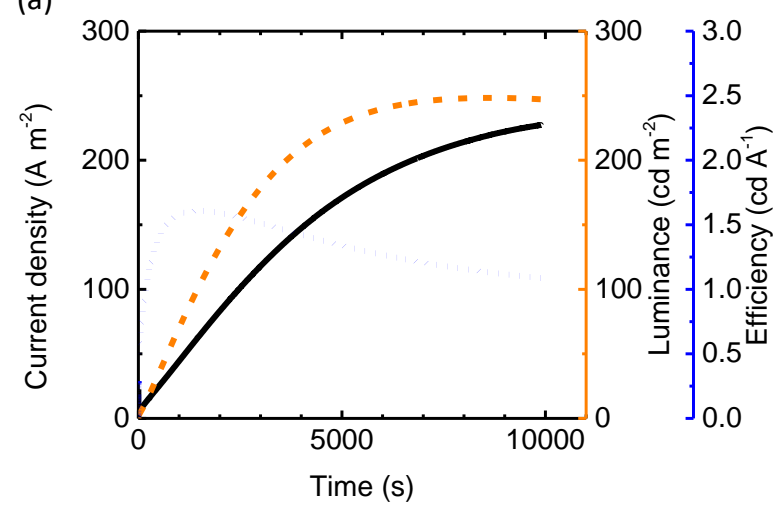

(b)

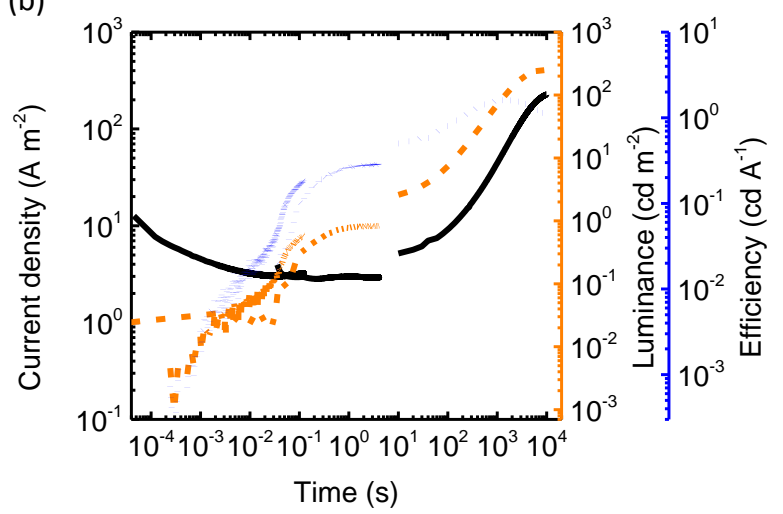

Figure 3. Experimentally determined transients of the current density (solid line), luminance (dashed lines) and efficiency (dotted lines) in an ITO/PEDOT:PSS/SY-PPV:PEO: $\mathrm{KCF}_{3} \mathrm{SO}_{3}$ $\left(=100: 20: 1\right.$, i.e. $\left.c 0=1.3 \cdot 10^{25} \mathrm{~m}^{-3}\right) / \mathrm{LiF} / \mathrm{Al}$ LEC with $L=230 \mathrm{~nm}$ and $V_{\text {bias }}=5 \mathrm{~V}$. (a) and (b) show the same results on linear and logarithmic scales respectively. 
WILEY-VCH

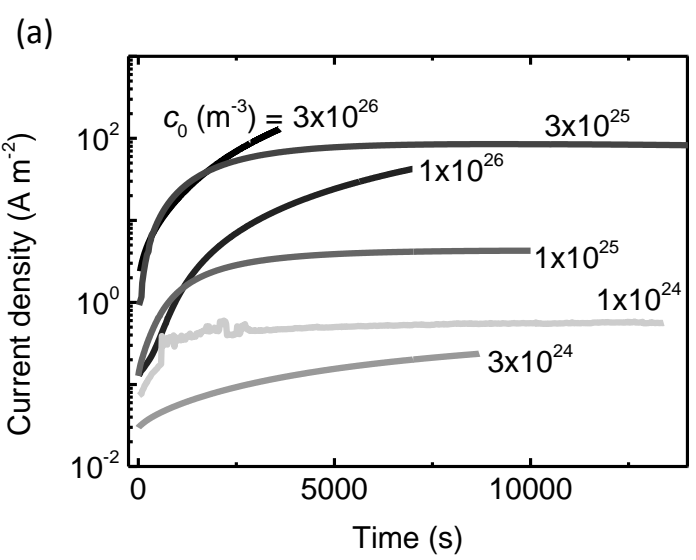

(b)

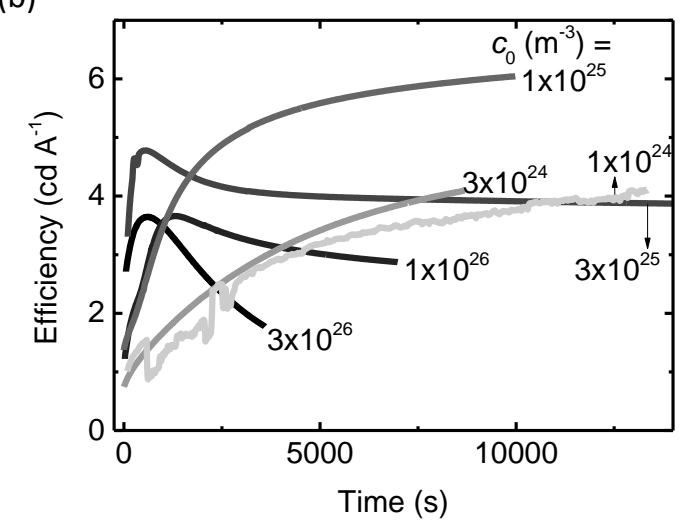

Figure 4. Experimentally determined transients of (a) the current density and (b) the efficiency of a ITO/PEDOT:PSS/SY-PPV:PEO(5:1)+ $\mathrm{KCF}_{3} \mathrm{SO}_{3} / \mathrm{LiF} / \mathrm{Al} \mathrm{LEC}$ with $L \approx 600 \mathrm{~nm}$ and $V_{\text {bias }}=6 \mathrm{~V}$. The admixed $\mathrm{KCF}_{3} \mathrm{SO}_{3}$ density $c_{0}$ is indicated at each graph. 
(a)

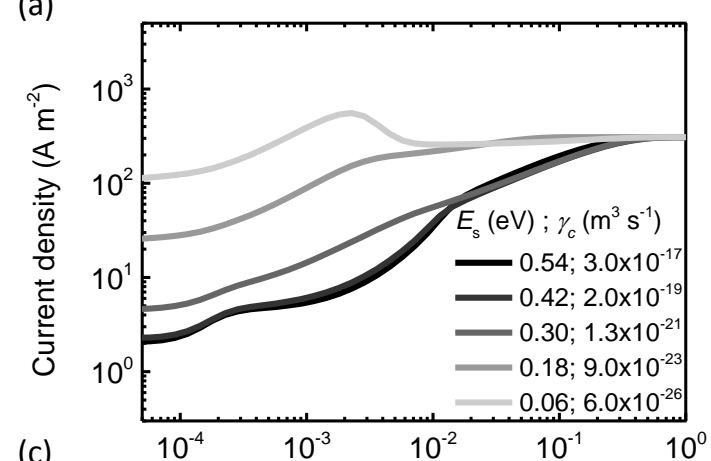

(c)

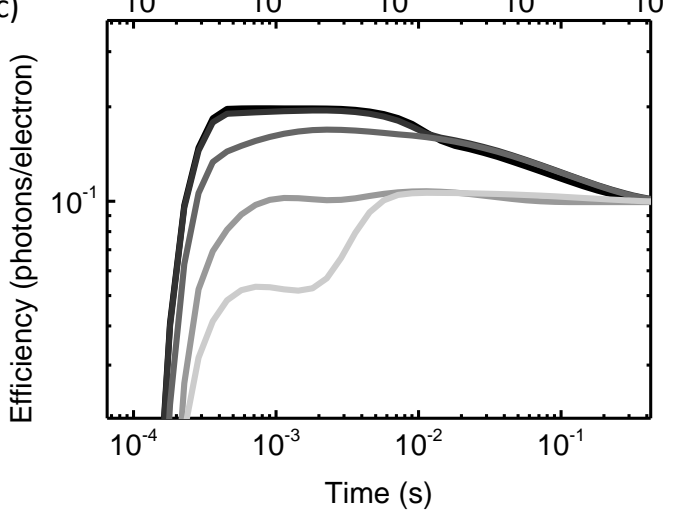

(b)

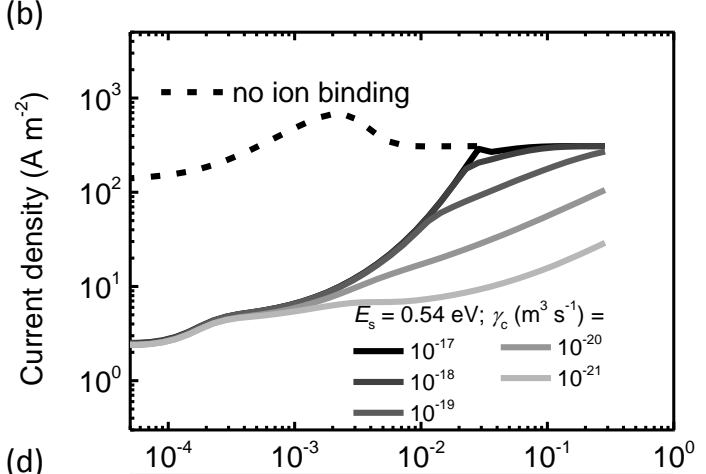

(d)

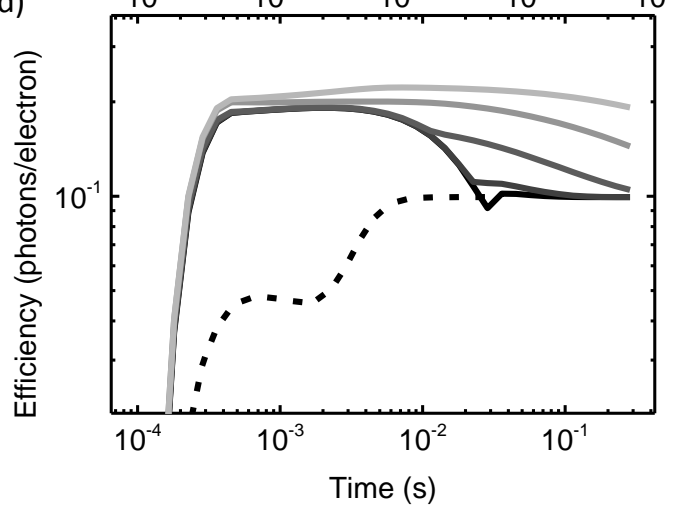

Figure 5. Calculated current density and efficiency transients for varying salt binding parameters (a), (c) $E_{\mathrm{s}}$ and $\gamma_{c}\left(\gamma_{c}\right.$ was varied in such a way to maintain a constant salt dissociation coefficient, $\gamma_{d}=\gamma_{c} \cdot \exp \left(E_{\mathrm{S}} / k T\right)$, which essentially results in the majority of salt dissociation to occur between $10^{-3}<t<10^{-1}$ ) and (b), (d) $\gamma_{c}$ as indicated in the legend. The dashed lines represent a calculation where no associated salt complex can be formed. The salt density is $3 \cdot 10^{25} \mathrm{~m}^{-3}$ and the applied bias voltage $3.5 \mathrm{~V}$. 


\section{WILEY-VCH}
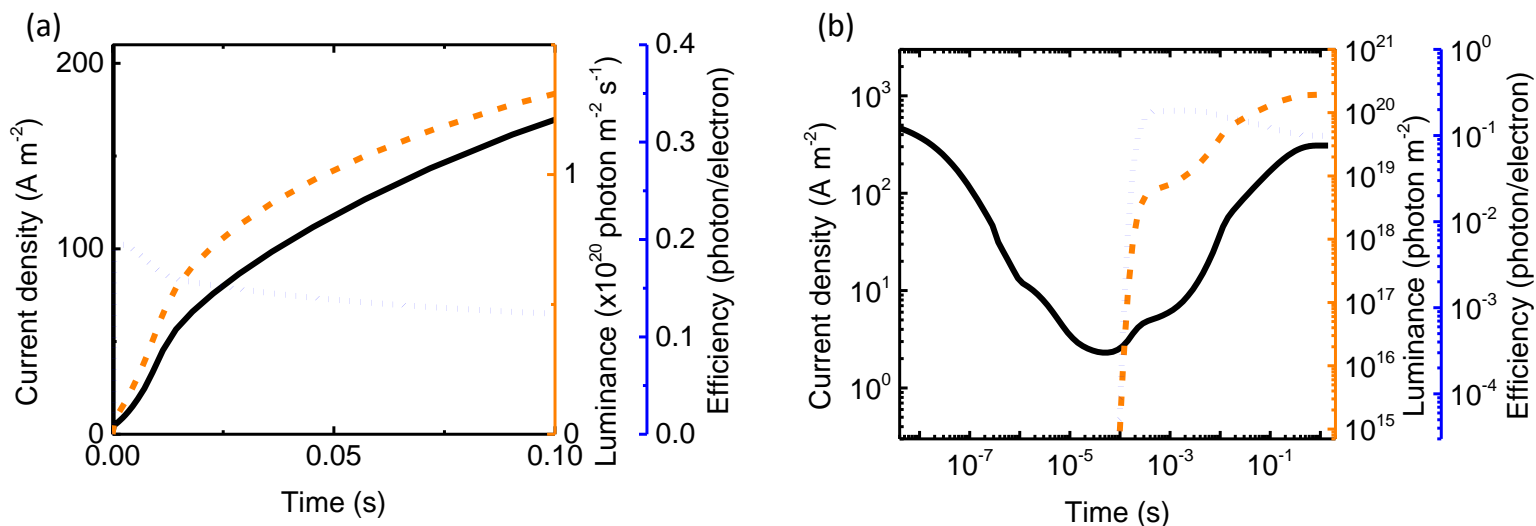

Figure 6. Calculated transients of the current density (solid), luminance (dashed) and efficiency (dotted) in a modeled LEC with ohmic contacts, an $L=100 \mathrm{~nm}, c_{0}=3 \cdot 10^{25} \mathrm{~m}^{-3}$, $V_{\text {bias }}=3.5 \mathrm{~V}, \mu_{\text {ion }}=10^{-12} \mathrm{~m}^{2} \mathrm{~V}^{-1} \mathrm{~s}^{-1}, \mu_{\mathrm{p} / \mathrm{n}}=10^{-11} \mathrm{~m}^{2} \mathrm{~V}^{-1} \mathrm{~s}^{-1}, E_{\mathrm{s}}=0.42 \mathrm{eV}$ and $\gamma_{c}=3 \cdot 10^{-17} \mathrm{~m}^{3} \mathrm{~s}^{-1}$. (a) and (b) show the same results on linear and logarithmic scales respectively. 


\section{WILEY-VCH}

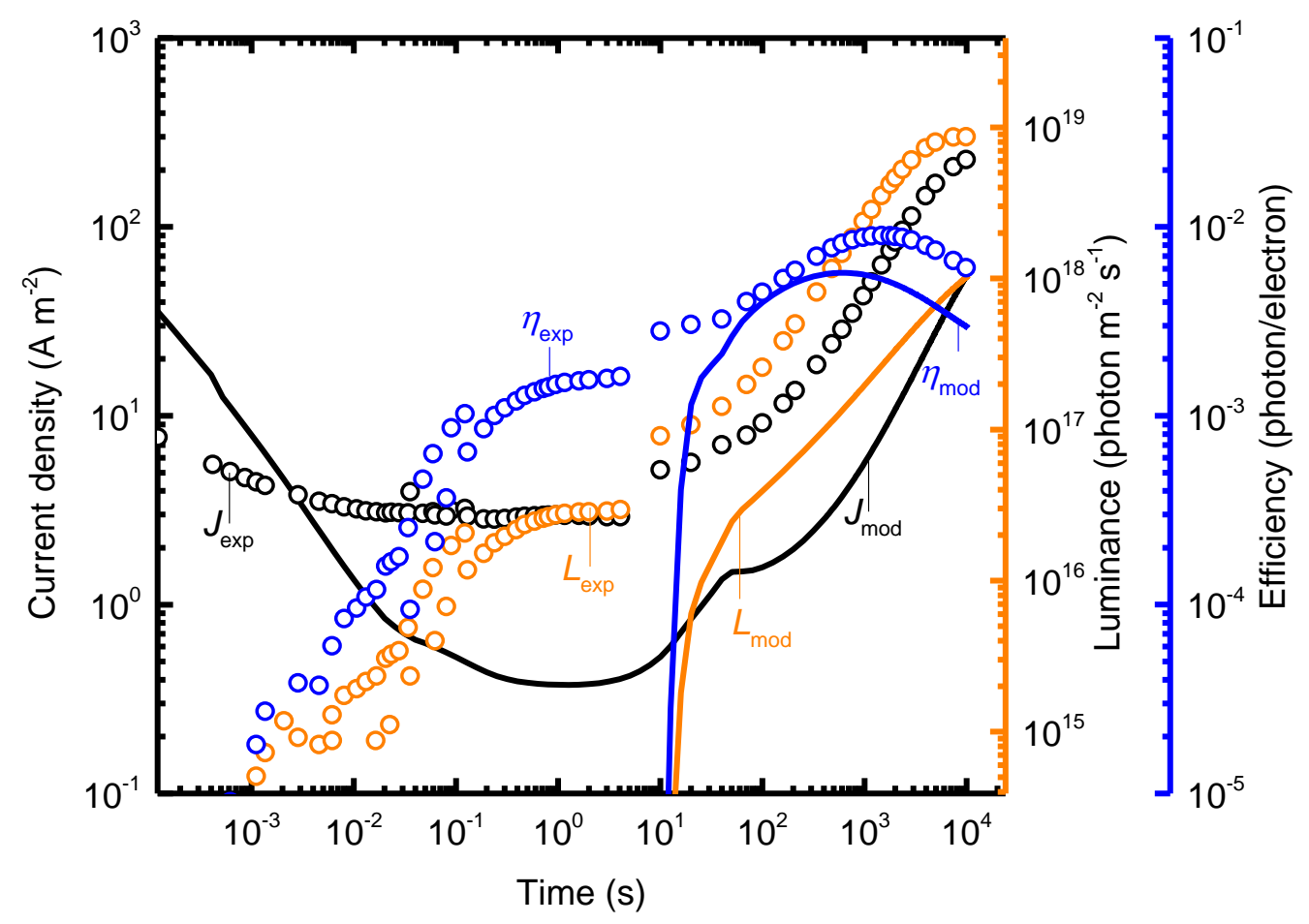

Figure 7. Calculated (lines) and experimental (symbols) transients of current (black), luminance (orange), and efficiency (blue) of 200 and $230 \mathrm{~nm}$ thick LECs biased at $5 \mathrm{~V}$. The time of the numerical data was corrected by a factor $2 \cdot 10^{3}$. 


\section{WILEY-VCH}

(a)

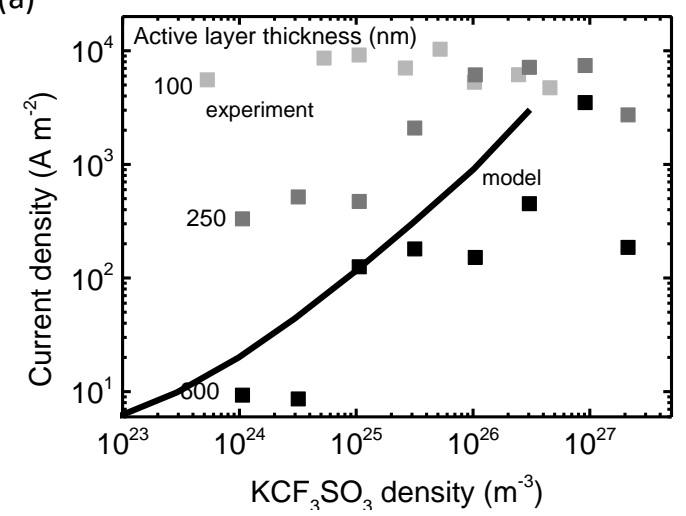

(b)

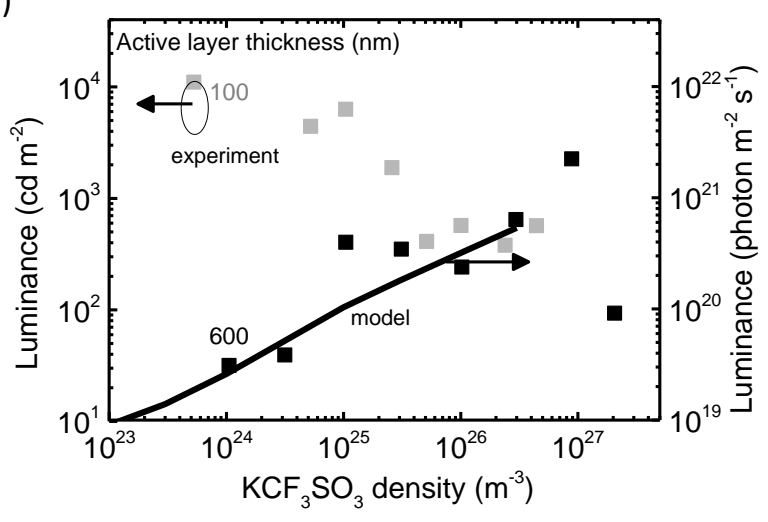


WILEY-VCH

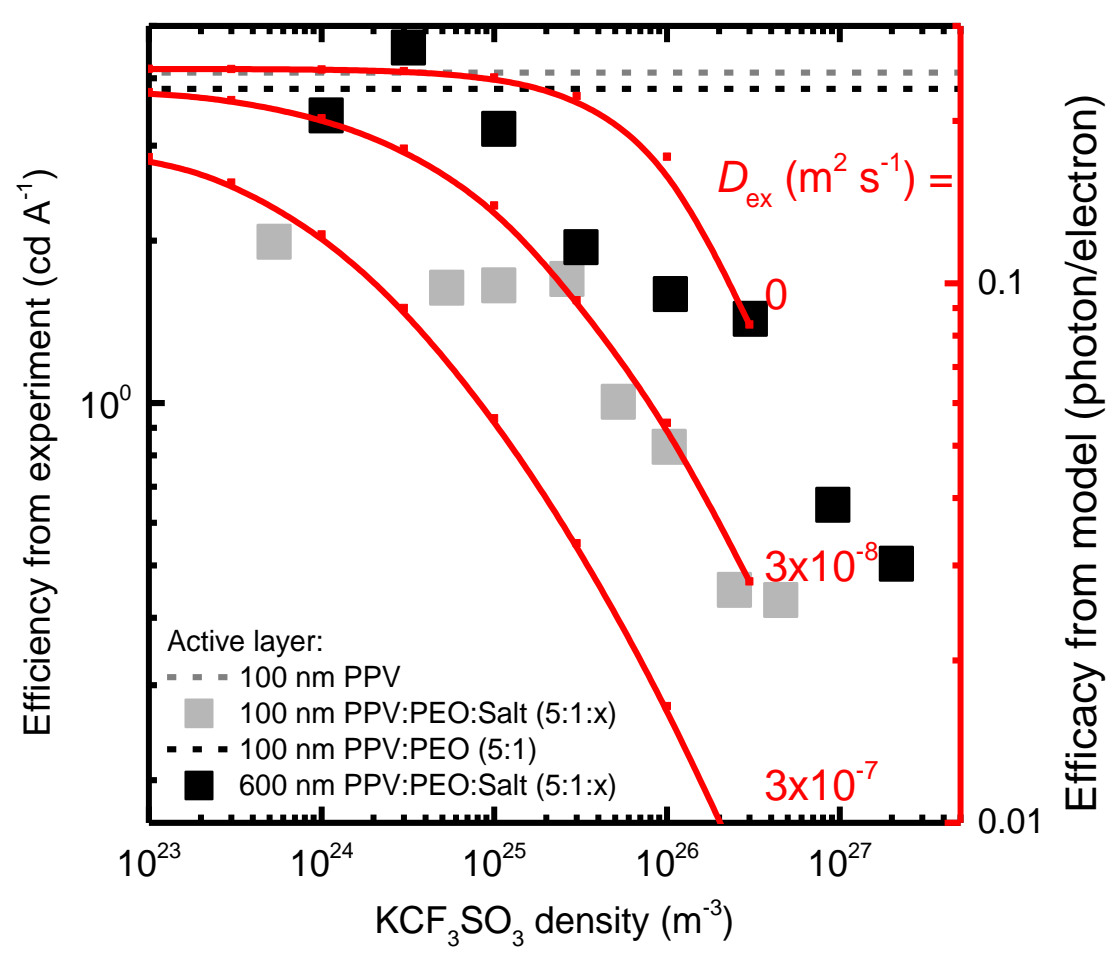




\section{WILEY-VCH}
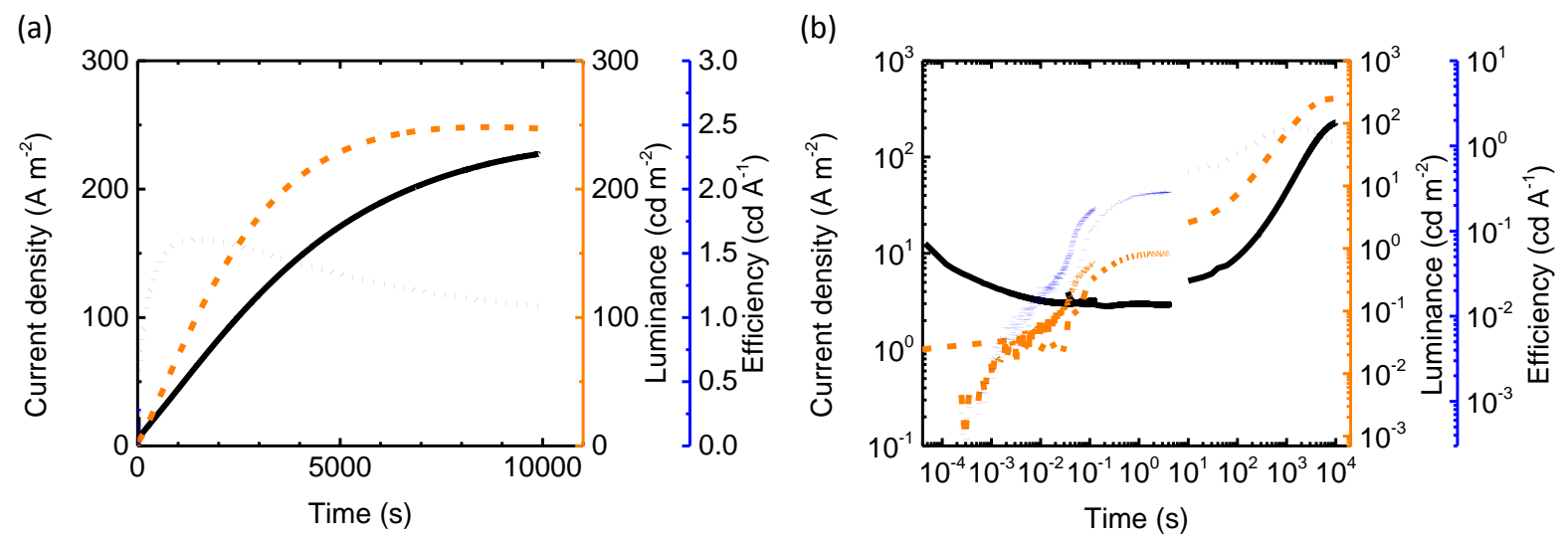


\section{WILEY-VCH}
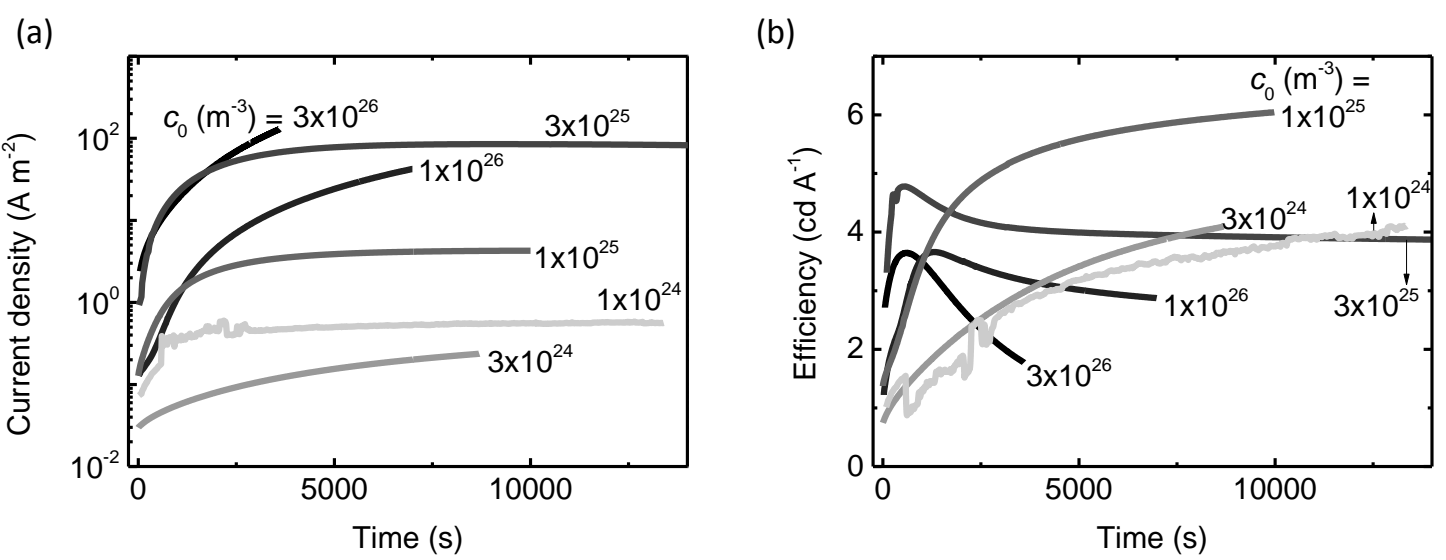


\section{WILEY-VCH}
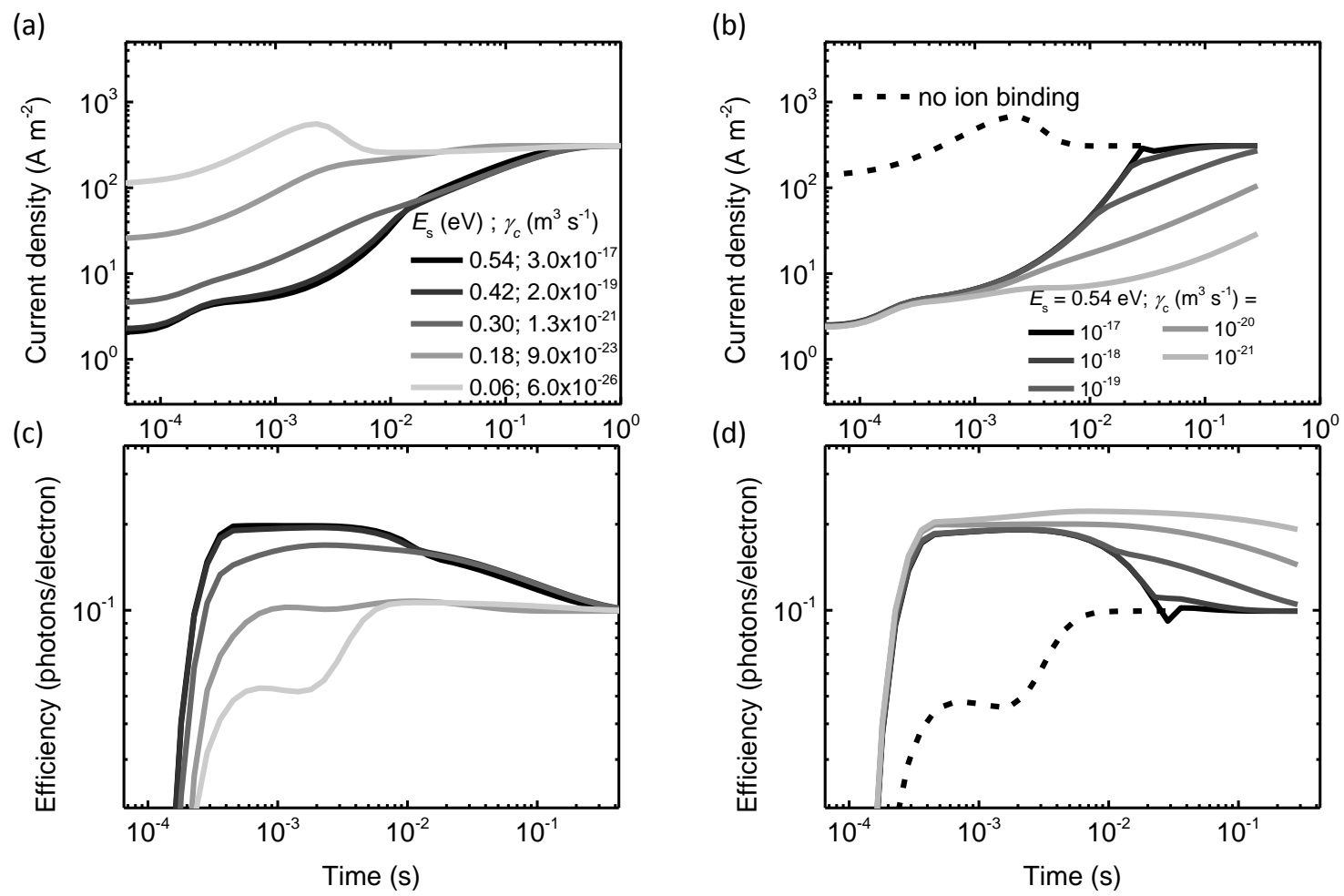


\section{WILEY-VCH}
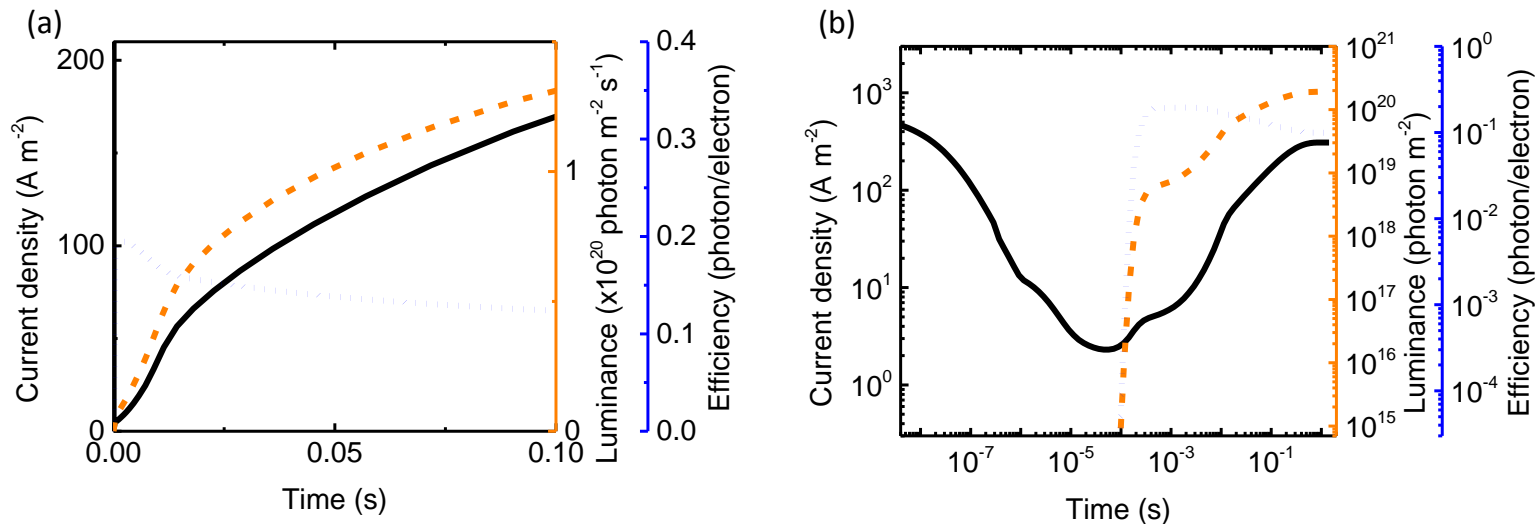


\section{WILEY-VCH}

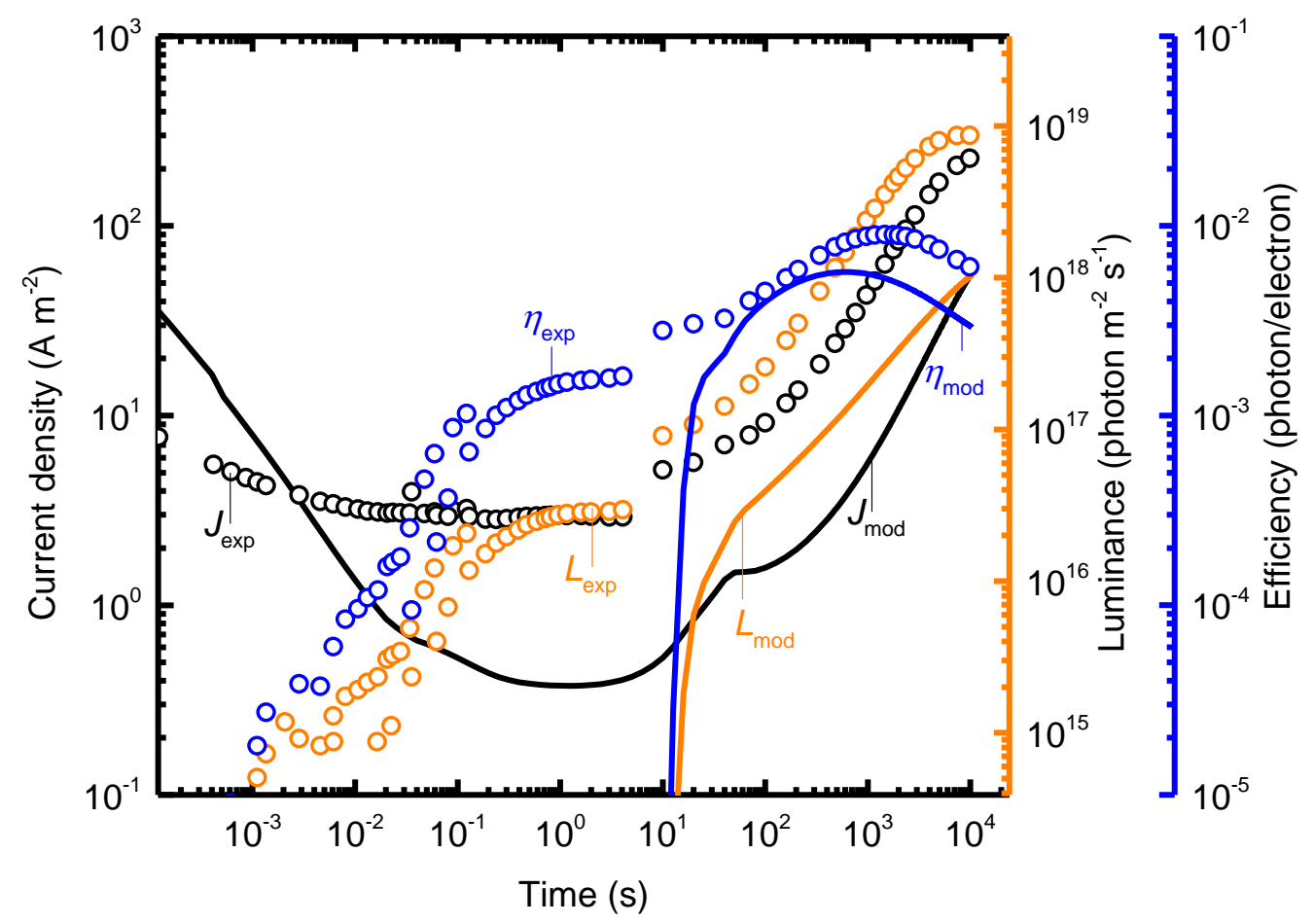


Copyright WILEY-VCH Verlag GmbH \& Co. KGaA, 69469 Weinheim, Germany, 2013.

\section{Supporting Information}

\section{Fundamental tradeoff between emission intensity and efficiency in light-emitting electrochemical cells}

\section{Stephan van Reenen, René A. J. Janssen, and Martijn Kemerink*}

\section{A. The numerical model to describe LEC operation}

To model the device operation of light-emitting electrochemical cells a numerical model is used which describes carrier injection, transport, and recombination in the active layer in the presence of mobile ions. ${ }^{1-3}$ This model solves the Boltzmann transport equations and Poisson's equation on a $2 \mathrm{D}$ grid by forward integration in time. In the next paragraphs a detailed description is given on the methods to describe charge transport, carrier injection and carrier recombination. Additional optional processes like i) a doping dependence in the carrier mobility, ii) a binding energy between the ions and iii) exciton quenching are included in the numerical model as well. Parameters that need to be defined for each calculation are shown in Table S1.

Table S1 Input parameters for the numerical model of a light-emitting electrochemical cell.

\begin{tabular}{|l|c|}
\hline Parameter & Symbol \\
\hline Mobility (electron/hole/anion/cation) & $\mu_{\mathrm{n}} / \mu_{\mathrm{p}} / \mu_{\mathrm{a}} / \mu_{\mathrm{c}}$ \\
\hline HOMO and LUMO of semiconductor & $E_{\text {HOMO }} / E_{\mathrm{LUMO}}$ \\
\hline Density of states of semiconductor & $N_{0}$ \\
\hline Salt density & $c_{0}$ \\
\hline Work function of contacts & $\varphi$ \\
\hline Drain voltage & $V_{\text {bias }}$ \\
\hline Temperature & $T$ \\
\hline Relative dielectric constant & $\varepsilon_{\mathrm{r}}$ \\
\hline
\end{tabular}

\section{A.1 Charge transport}

In the 2D model a rectangular grid is used with grid points at the corners of each cell. The areas of the cells are determined by the widths of the columns and rows which can be specified per row or column. For each cell a material type is specified, either gate, contact, dielectric or semiconductor. Calculations of current, recombination, etc. are performed on the grid points. The 4 cells surrounding the grid point are in equilibrium with each other, which is effectuated by area-average weighting of these 4 cells. Consequently, a single quasi Fermi level is defined on each grid point.

Carrier transport is described by the following Boltzmann transport equations:

$$
J_{i}=-q \mu_{i} n_{i} \nabla \psi_{F, i}
$$

where $q$ is the elementary charge, $\mu_{i}$ and $n_{\mathrm{i}}$ are the mobility and density for electrons $(i=n)$, holes $(i=p)$, anions $(i=a)$, or cations $(i=c)$ and $\psi_{F, i}$ is the quasi Fermi energy which is given by

$$
\psi_{F, n}=\psi_{L}-\frac{k T}{q} \ln \frac{n_{n}}{N_{0}}
$$




\section{WILEY-VCH}

$$
\begin{aligned}
& \psi_{F, p}=\psi_{H}+\frac{k T}{q} \ln \frac{n_{p}}{N_{0}}, \\
& \psi_{F, a}=V-\frac{k T}{q} \ln \frac{n_{a}}{N_{0}}, \\
& \psi_{F, c}=V+\frac{k T}{q} \ln \frac{n_{c}}{N_{0}},
\end{aligned}
$$

where $\psi_{L}=E_{L U M O}+V$ and $\psi_{H}=E_{\text {HOMO }}+V$ and $V$ is the electrostatic potential. $k$ is Boltzmann's constant and $T$ is the temperature. Einstein's relation between the diffusion constant and the mobility is assumed to hold. The ion current is zero at the electrodes, for the electrodes are ionically blocking. The continuity equations are

$$
\begin{aligned}
& \frac{d n}{d t}=\frac{1}{q} \frac{d J_{n}}{d x}-R, \\
& \frac{d p}{d t}=-\frac{1}{q} \frac{d J_{p}}{d x}-R, \\
& \frac{d a}{d t}=\frac{1}{q} \frac{d J_{a}}{d x}, \\
& \frac{d c}{d t}=-\frac{1}{q} \frac{d J_{c}}{d x},
\end{aligned}
$$

for electrons, holes, anions and cations, respectively. Here $J_{\mathrm{i}}$ is the current density of particle $i$ and $R$ is the recombination rate. The potential profile throughout the device is calculated from Poisson's equation:

$$
\nabla^{2} V=-\frac{q}{\varepsilon_{0} \varepsilon_{r}}(p-n+c-a)
$$

where $\varepsilon_{0}$ and $\varepsilon_{\mathrm{r}}$ are the dielectric and the relative dielectric constant, respectively.

\section{A.2 Carrier injection}

To describe the injection of electrons and holes into the semiconductor, the following parameters are of importance: the HOMO and LUMO levels of the semiconductor; the Fermi level of the contacts; the grid-point spacing at the interfaces; and the charge carrier density in the semiconductor at the interface. The injection model used for calculations reported in the main article is based on Boltzmann injection.

A relatively short grid-point spacing is needed close to the electrodes to avoid under-sampling. Under-sampling at the interfaces namely leads to an unphysical reduction of the charge carrier injection rate for alternative models based on Fowler-Nordheim tunneling and EmtageO'Dwyer injection models. It namely increases the distance to be overcome by the injected carriers to reach the first grid-point after the contact. Evasion of under-sampling at the interfaces by use of a small grid-point spacing leads to extremely long calculation times: this time scales with one over the smallest spacing of the numerical mesh to maintain numerical stability. In optimized LECs ${ }^{2}$ injection, however, does not limit the current through the LEC. deMello, ${ }^{4}$ for instance, solved this issue by making use of an adaptive-grid method-of-lines. Here another solution was chosen by designing another injection model based on Boltzmann carrier injection. It uses a Boltzmann factor to describe injection in the following manner taking into account the band bending just behind the first grid-point:

$$
n(1)=N_{0} \exp \left(-\frac{q \varphi_{n}-q[V(2)-V(1)]}{k T}\right) \text {, }
$$

where $n(1)$ is the electron density at the first grid-point next to the electrode, $\varphi_{n}$ is the injection barrier height, and $V(i)$ the potential at grid-point $i(=1,2)$. Hence, the Boltzmann 


\section{WILEY-VCH}

factor is effectively evaluated at the $2^{\text {nd }}$ grid point. The potential at the first grid point $V(1)$ is taken as a boundary condition for the potential and is thus constant, i.e. $0+\varphi_{n}$ or $V_{\text {bias }}+\varphi_{n}$. The potential at the neighboring grid-point however changes due to space-charge accumulated at the EDLs which facilitates injection of electron or holes. In this model injection is not affected by the grid-point spacing and thus thicker devices can be simulated while not having to alter the number of grid-points because of injection problems.

\section{A.3 Doping dependent carrier mobility}

For the implementation of a doping dependence in the charge carrier mobility experimental data was parameterized and implemented in the model. The doping dependence of the carrier mobility in PPV (see Figure S1b) ${ }^{5}$ can be described by the following equation:

$$
\mu_{p / n}(f)=\mu_{p / n, 0}\left(A+B \cdot(f+C)^{2}\right),
$$

where $A, B$ and $C$ are the parameters. In Figure S1a a plot of this model is shown for $A=-$ $1 \cdot 10^{-11}, B=7.2 \cdot 10^{-10}$ and $C=0.167$, as well as the doping density dependence measured in Super yellow PPV in Ref. 5.

(a)

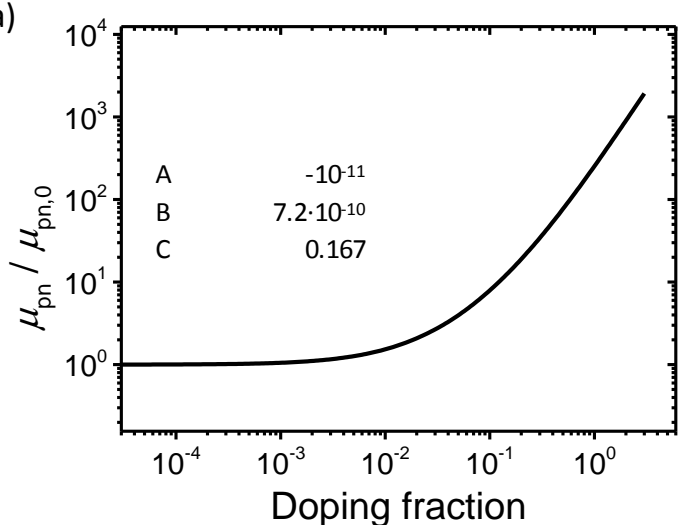

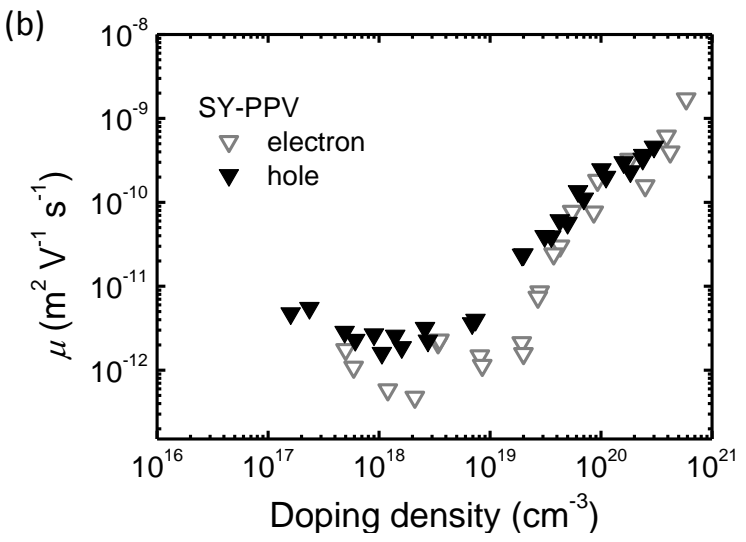

Figure S1 The electron/hole mobility as a function of the doping fraction or density. Experimentally determined graphs are shown (d) for Super Yellow PPV. ${ }^{5}$ The model graph is shown in (a) based on Equation Fel! Hittar inte referenskälla.) for the parameters in the inset table.

\section{A.4 Ion binding}

The binding energy between anions and cations was taken into account by introduction of a $5^{\text {th }}$ type of particle: the salt molecule. The binding energy between anion and cation is $E_{\mathrm{s}}(>0)$. The total anion-cation binding capture rate $C_{\mathrm{i}}\left(\mathrm{m}^{-3} \mathrm{~s}^{-1}\right)$ at each grid point $i$ is

$$
C_{i}=\gamma_{c} \cdot\left(n_{a, i}-n_{p, i}\right) \cdot\left(n_{c, i}-n_{n, i}\right)
$$

with $\gamma_{c}$ the capture coefficient $\left(\mathrm{m}^{3} \mathrm{~s}^{-1}\right)$ and $n_{\mathrm{x}, \mathrm{i}}$ the density at grid point $i$ for anions $(x=a)$, cations $(x=c)$, holes $(x=p)$ and electrons $(x=n)$. Note that ions compensated by electrons or holes do not contribute to formation of salt. For the dissociation of the salt the detrapping rate $E_{\mathrm{i}}\left(\mathrm{m}^{-3} \mathrm{~s}^{-1}\right)$ was used:

$$
E_{i}=K \cdot \gamma_{d} \cdot n_{s}
$$

where $n_{\mathrm{s}}$ is the salt density, $K$ is the mass action law constant (here taken $10^{27} \mathrm{~m}^{-3}$ ) and the coefficient $\gamma_{d}$ is related to the capture coefficient $\gamma_{c}$ via detailed balance:

$$
\gamma_{d}=\gamma_{c} \cdot \exp \left(-\frac{E_{s}}{k T}\right) \text {. }
$$

Finally, the net ion binding rate $U_{\mathrm{i}}\left(\mathrm{m}^{-3} \mathrm{~s}^{-1}\right)$ is given by 


\section{WILEY-VCH}

$$
U_{i}=C_{i}-E_{i}
$$

Before each simulation the device was allowed to reach steady-state at $V_{\text {bias }}=0 \mathrm{~V}$ to allow salt to form.

\section{A.5 Exciton formation and decay}

Electrons and holes recombine following a Langevin process in this model:

$$
R=\frac{q\left(\mu_{n}+\mu_{p}\right)}{\varepsilon_{0} \varepsilon_{r}} p n \text {. }
$$

To calculate the efficiency and luminance of the LEC, also the decay of excitons is included. To do so, recombination of electrons and holes give rise to singlet and triplet excitons according to a fraction $r_{\mathrm{S}}$ and $r_{\mathrm{T}}$. In addition exciton diffusion with diffusion constant $D_{\mathrm{ex}, \mathrm{S} / \mathrm{T}}$ is included. For a given lifetime of the singlet and triplet excitons, $\tau_{\mathrm{S}}$ and $\tau$ T, the singlet/triplet rate equations become

$$
\frac{\partial S}{\partial t}=r_{S} R-\frac{S}{\tau_{S}}-D_{e x, S} \frac{d^{2} S}{d x^{2}}
$$

and

$$
\frac{\partial T}{\partial t}=\left(1-r_{S}\right) R-\frac{S}{\tau_{T}}-D_{e x, T} \frac{d^{2} T}{d x^{2}}
$$

where $S$ and $T$ are the singlet and triplet exciton densities.

To implement singlet exciton quenching by polarons, a model must be used to describe the density dependence of the quenching rate $k_{\mathrm{PQ}}$. The exciton quenching can then be implemented by the definition of

$$
\frac{1}{\tau_{S}}=\frac{1}{\tau_{S, 0}}+k_{P Q}(n+p)
$$

where $\tau_{S, 0}$ is the singlet exciton lifetime in absence of any quenchers. As the numerical model does not use the molecular sites for calculation but a rectangular grid, quenching models like Förster resonance energy transfer cannot be simply implemented. The method to implement exciton quenching by polarons is by use of obtained experimental data. The doping dependence of $k_{F L} /\left(k_{F L}+k_{P Q}\right)$ is measured as shown in Ref [5], where $k_{\mathrm{FL}}=1 / \tau_{S, 0}$. From this $k_{\mathrm{PQ}}(n+p)$ can be determined, parameterized and put into the model to give the quenching rate at each cell at a given polaron density. Excitons on site $i$ do not interact with excitons on site $j \neq i$. Long range interactions are only mediated by the diffusion of excitons to another cell. As the time steps in the calculation for charge transport are typically orders of magnitude larger than the lifetime of the excitons, $k_{F L}$ and $D_{\text {ex }}$ are reduced by a factor $10^{-5}$, which slows down both processes equally. Such a delay may give errors in the calculation of the transient behavior of the exciton decay processes. For transients which are limited by the movement of ions care is taken that the ion diffusion coefficient $D_{\text {ion }}=\mu_{\text {ion }} k T / q$ remains smaller than $D_{\text {ex }}$ by at least a factor 10 to minimize this effect.

\section{A.6 Model operation}

In this paragraph the general operation of the model is described. Initially the device only contains anions and cations which electrostatically compensate each other. No electrons or holes are present. Only at the contacts electrons and holes are present due to the boundary conditions imposed by the chosen injection model. In addition, the bias voltage is applied to one of the contacts, whereas the other contact is set at $0 \mathrm{~V}$. For a defined number and size of time steps, the model then calculates the Fermi energy of all the carriers, solves the Boltzmann transport equations, accounts for electron-hole recombination, and solves the 


\section{WILEY-VCH}

continuity equations to determine the new carrier densities at each grid point. A steady-state solution is obtained when the current, which is determined by the sum of current through the contacts, becomes constant. 


\section{B. effect of electrodes and PPV:electrolyte ratio on the operation of LECs}

LECs were prepared with varying PPV:electrolyte ratios and contacting electrodes, followed by electrical characterization of the quasi-steady state characteristics. The results are shown in Figure B.1. For the ITO-blend-Al devices at low salt concentrations, a relatively low current density is observed as compared to the ITO-PEDOT-blend-LiF-Al devices, which can be attributed to poor injection due to large injection barriers and the absence of sufficient salt to form electric double layers. At high salt concentrations, the efficiency of the ITO-PEDOTblend-LiF-Al devices is somewhat larger compared to the ITO-blend-Al devices which may be attributed to an improved balance in carrier injection.

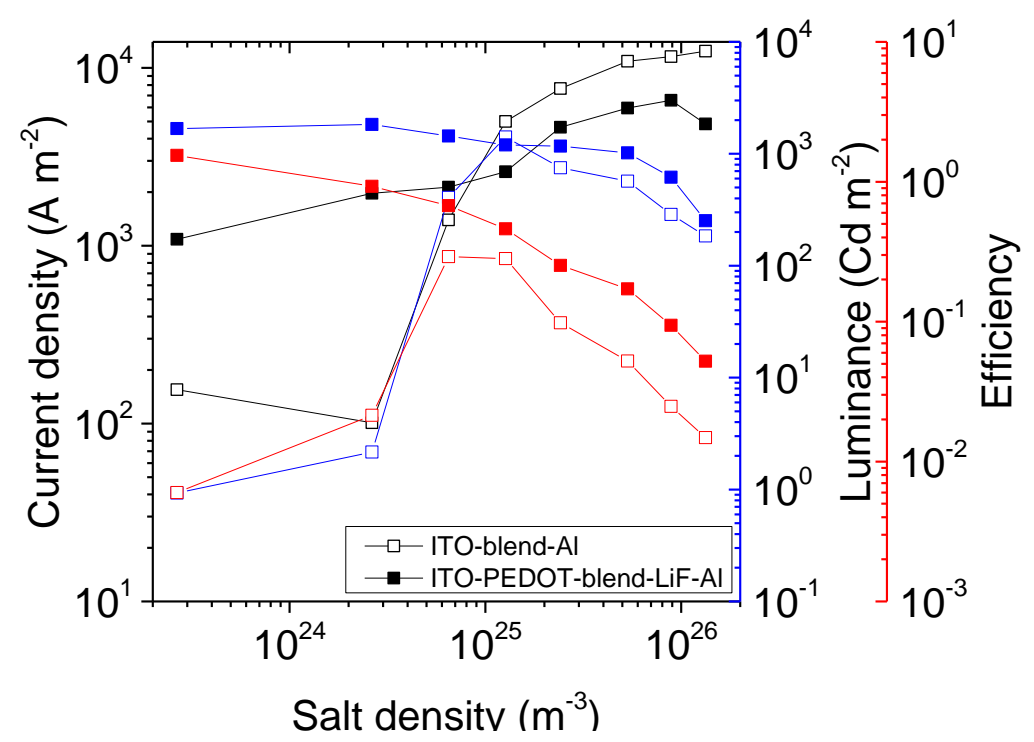

Figure B.1. Experimentally (symbols, $V_{\text {bias }}=8 \mathrm{~V}$ ) determined quasi-steady-state current density (black), luminance (blue), and efficiency (red) of LECs with varying active layer blends with a similar thickness of roughly $100 \mathrm{~nm}$. The active layer blends consisted of $\mathrm{PPV}: \mathrm{PEO}+\mathrm{KCF}_{3} \mathrm{SO}_{3}\left(\mathrm{PEO}: \mathrm{KCF}_{3} \mathrm{SO}_{3}=5: 1\right)$ with ratios of $1: x$ with $\mathrm{x}=(1,0.5,0.25,0.1,0.05$, $0.025,0.01,0.001)$. 


\section{C. additional results belonging to Figure 5}

(a)

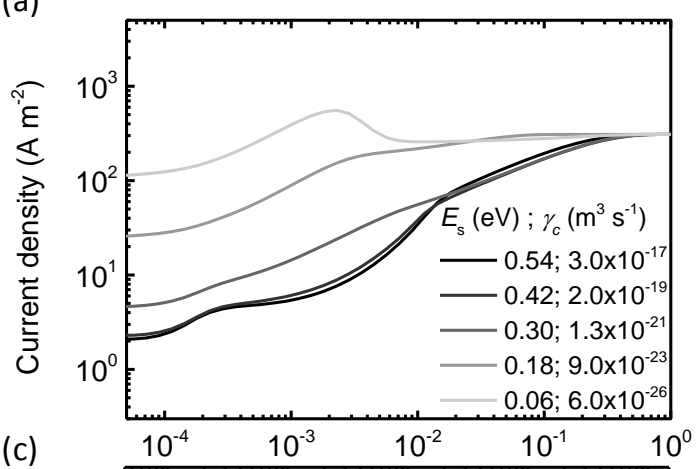

(c)

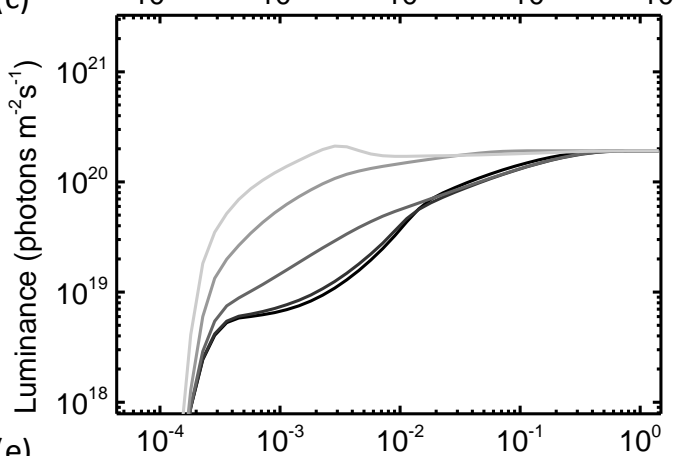

(e)

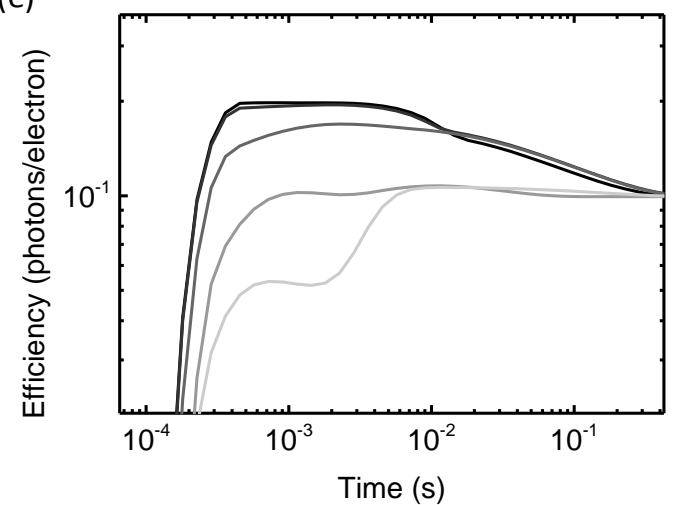

(b)

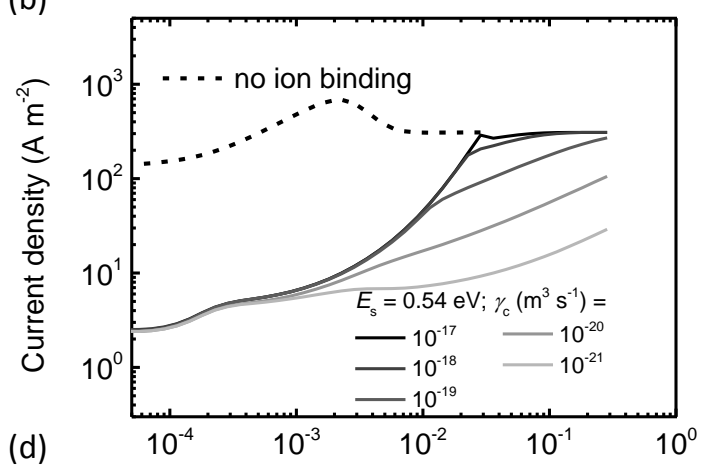

(d)

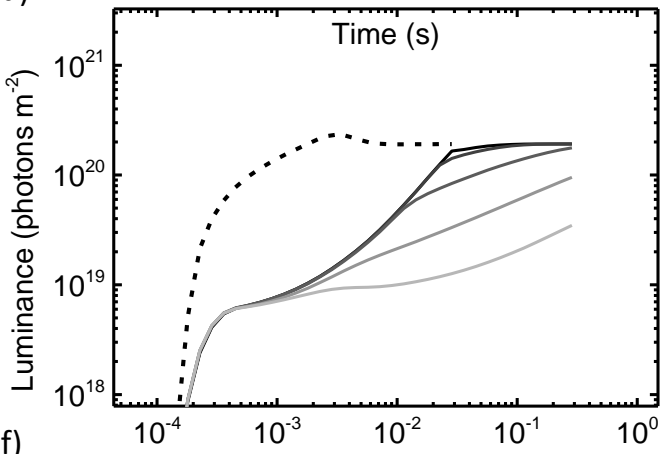

(f)

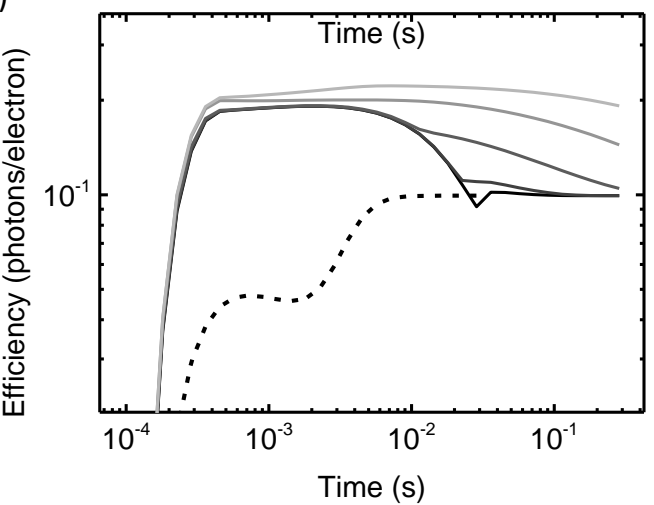

Figure C.1. Calculated current density, luminance and efficiency transients for varying salt binding parameters (a), (c), (e) $E_{\mathrm{s}}$ and $\gamma_{c}\left(\gamma_{c}\right.$ was varied in such a way to maintain a constant salt dissociation coefficient, $\gamma_{d}=\gamma_{c} \cdot \exp \left(E_{\mathrm{S}} / k T\right)$, which essentially results in the majority of salt dissociation to occur between $10^{-3}<t<10^{-1}$ ) and (b), (d), (f) $\gamma_{c}$ as indicated in the legend. The dashed lines represent a calculation where no associated salt complex can be formed. The salt density is $3 \cdot 10^{25} \mathrm{~m}^{-3}$ and the applied bias voltage $3.5 \mathrm{~V}$. 


\section{WILEY-VCH}

\section{Parameter choice to achieve qualitative fit of current and efficiency transients in LECs}

The mobility of the electrons and holes in absence of electrochemical doping is linearly related to the time at which electrochemical doping and luminance starts, at $t=10^{-4} \mathrm{~s}$ in Figure 6 for $\mu_{\mathrm{p} / \mathrm{n}}=10^{-11} \mathrm{~m}^{2} \mathrm{~V}^{-1} \mathrm{~s}^{-1}$. In Ref. ${ }^{5}$ it was shown by experiments that this mobility is roughly $10^{-12} \mathrm{~m}^{2} \mathrm{~V}^{-1} \mathrm{~s}^{-1}$ for SY-PPV. Here, such a mobility would result in an initial luminance at $t=10^{-3} \mathrm{~s}$, which is in line with the experimental data in Figure 3. Then, to achieve the correct steady-state current density a doping dependent mobility needs to be included (see the section A.3 of the Supporting Information), as well as a salt density of $5 \cdot 10^{25} \mathrm{~m}^{-3}$, similar to the experiments in Figure 3. The salt density is related to the final doping density throughout the doped regions. Finally parameters should be altered to move the transient doping process from $10^{-2}<t(\mathrm{~s})<10^{1}$ in Figure 6 to $10^{1}<t(\mathrm{~s})<10^{4}$ in Figure 3. In Ref. 3 we showed that for $\mu_{\text {ion }}<10 \mu_{\mathrm{p} / \mathrm{n}}$, which is the case here as well, the transient doping process is constrained by the less mobile ionic carriers. So the parameters to modify the time at which electrochemical doping takes place are the ion mobility and the ion capture coefficient. To achieve similarity to the experiment, the ion mobility and the ion capture coefficient $\left(\gamma_{c}\right)$ in the model need to be reduced. Now assume that anion-cation recombination is similar to electron-hole recombination and can be described by a Langevin process with a recombination coefficient equal to $2 q \mu_{\text {ion }} / \varepsilon_{0} \varepsilon_{r}=\gamma_{c}$. Then the time at which electrochemical doping takes place is only related to $\mu_{\text {ion, for }} \mu_{\text {ion }}<10 \mu_{\mathrm{p} / \mathrm{n}}$. An ion mobility in the order of $10^{-15} \mathrm{~m}^{2} \mathrm{~V}^{-1} \mathrm{~s}^{-1}$ and a corresponding $\gamma_{c}=9 \cdot 10^{-24} \mathrm{~m}^{3} \mathrm{~s}^{-1}$ likely result in the best fit of the data shown in Figure 3 . This mobility value is lower than found by impedance spectroscopy $\left(10^{-13} \mathrm{~m}^{2} \mathrm{~V}^{-1} \mathrm{~s}^{-1}\right.$ in Ref. 6) or modeling of transient operation of LECs in planar device configuration at $T=323 \mathrm{~K}\left(5 \cdot 10^{-}\right.$ ${ }^{11} \mathrm{~m}^{2} \mathrm{~V}^{-1} \mathrm{~s}^{-1}$ in Ref. 3). In the experiments described in these references, a PPV:PEO ratio of 1:1.35 was used as opposed to the 1:0.2 used in the experiments here. This reduction in PEO concentration is likely to result in a strong reduction of the ion mobility, possibly to $10^{-15} \mathrm{~m}^{2}$ $\mathrm{V}^{-1} \mathrm{~s}^{-1}$ which is needed here to fit the experimental data. 


\section{WILEY-VCH}

\section{E. conversion factors for experimental luminance and efficiency}

To determine the conversion factors from $\mathrm{cd} \mathrm{m} \mathrm{m}^{-2}$ to photon $\mathrm{m}^{-2} \mathrm{~s}^{-1}$, the process of electroluminescence is considered, starting from a given electrical current until the luminance in $\mathrm{cd} \mathrm{m}^{-2}$.

The current density $J$ corresponds to a number $J / q\left(\mathrm{~m}^{-2} \mathrm{~s}^{-1}\right)$ of electrons. These carriers recombine with a recombination efficiency $\eta_{\text {rec }}$ (assumed to be 1 in LECs) into excitons. These excitons can decay radiatively with an efficiency $\eta_{\mathrm{rad}}$ which is based on the singlet triplet ratio (in this manuscript a singlet emitter is considered, rendering 25\%) and the PL

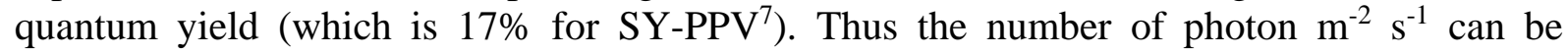
determined from the current density by $J_{\text {photon }}=J \cdot \eta_{\mathrm{rec}} \cdot \eta_{\mathrm{rad}} / q$.

To convert this number (in photon $\mathrm{m}^{-2} \mathrm{~s}^{-1}$ ) into illuminance $E_{\mathrm{v}}$ (in $1 \mathrm{~m} \mathrm{~m}^{-2}$ ), the photon energy, the photon intensity and the eye sensitivity curve $K(\lambda)$ must be taken into account by the following integration:

$$
E_{v}=\int_{0}^{\infty} K(\lambda) \cdot \frac{h c}{\lambda} \cdot \frac{P L(\lambda)}{\int_{0}^{\infty} P L(\lambda) d \lambda} \cdot J_{\text {photon }} d \lambda
$$

where $h$ is Planck's constant, $c$ the speed of light, $\lambda$ the wavelength and $P L(\lambda)$ the emission spectrum of SY-PPV.

From the illuminance, the luminance can be determined by considering all light emitted in all directions comprising a hemisphere: $E_{\mathrm{v}} / 2 \pi=L$ in $\mathrm{cd} \mathrm{m}^{-2}$.

From the given equations, conversion factors can now be determined to convert $\mathrm{cd} \mathrm{m}^{-2}$ into photon $\mathrm{m}^{-2} \mathrm{~s}^{-1}$, i.e. $3.5 \cdot 10^{16}$, as well as $\mathrm{cd} \mathrm{A}^{-1}$ into photon per electron, i.e. 0.0056 . 


\section{WILEY-VCH}

\section{F. References}

[1] S. van Reenen, P. Matyba, A. Dzwilewski, R. A. J. Janssen, A. Edman, M. Kemerink, Adv. Funct. Mater. 2011, 21, 1795.

[2] S. van Reenen, P. Matyba, A. Dzwilewski, R. A. J. Janssen, L. Edman, M. Kemerink, J Am Chem Soc 2010, 132, 13776.

[3] S. van Reenen, R. A. J. Janssen, M. Kemerink, Org. Electron. 2011, 12, 1746.

[4] J. C. deMello, Phys. Rev. B 2002, 66, 235210.

[5] S. van Reenen, M. V. Vitorino, S. C. J. Meskers, R. A. J. Janssen, M. Kemerink, Phys. Rev. B 2014, 89, 205206.

[6] S. van Reenen, R. A. J. Janssen, M. Kemerink, Adv. Funct. Mater. 2012, 22, 4547.

[7] E. W. Snedden, L. A. Cury, K. N. Bourdakos, A. P. Monkman, Chem. Phys. Lett. 2010, 490, 76. 